PREPARED FOR THE U.S. DEPARTMENT OF ENERGY, UNDER CONTRACT DE-AC02-76CH03073

PPPL-3931rev

PPPL-3931rev

UC-70

MHD-induced Energetic lon Loss during H-mode Discharges in the National Spherical Torus Experiment (NSTX)

by

S.S. Medley, N.N. Gorelenkov, R. Andre, R.E. Bell, D.S. Darrow,

E.D. Fredrickson, S.M. Kaye, B.P. LeBlanc, A.L. Roquemore, and the NSTX Team

Revised June 2004

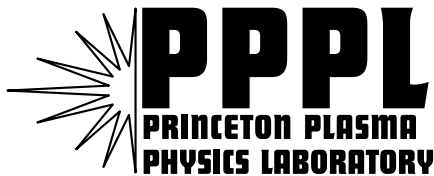

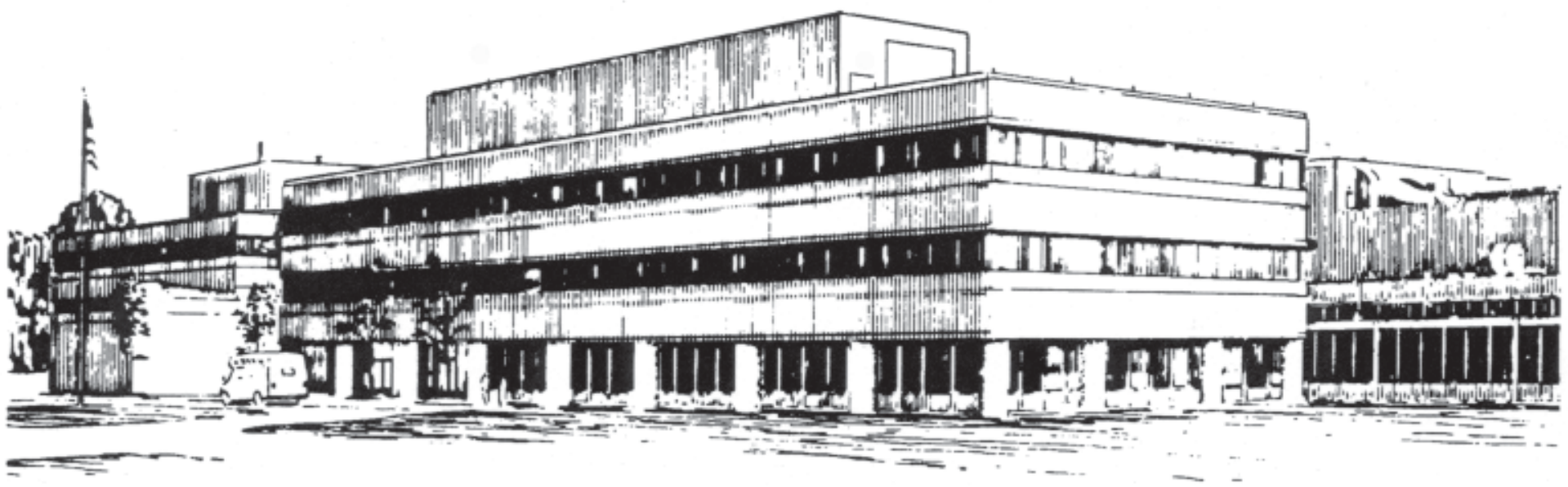

PRINCETON PLASMA PHYSICS LABORATORY PRINCETON UNIVERSITY, PRINCETON, NEW JERSEY 


\section{PPPL Reports Disclaimer}

This report was prepared as an account of work sponsored by an agency of the United States Government. Neither the United States Government nor any agency thereof, nor any of their employees, makes any warranty, express or implied, or assumes any legal liability or responsibility for the accuracy, completeness, or usefulness of any information, apparatus, product, or process disclosed, or represents that its use would not infringe privately owned rights. Reference herein to any specific commercial product, process, or service by trade name, trademark, manufacturer, or otherwise, does not necessarily constitute or imply its endorsement, recommendation, or favoring by the United States Government or any agency thereof. The views and opinions of authors expressed herein do not necessarily state or reflect those of the United States Government or any agency thereof.

\section{Availability}

This report is posted on the U.S. Department of Energy's Princeton Plasma Physics Laboratory Publications and Reports web site in Fiscal Year 2004. The home page for PPPL Reports and Publications is: http://www.pppl.gov/pub_report/

DOE and DOE Contractors can obtain copies of this report from:

U.S. Department of Energy

Office of Scientific and Technical Information

DOE Technical Information Services (DTIS)

P.O. Box 62

Oak Ridge, TN 37831

Telephone: (865) 576-8401

Fax: (865) 576-5728

Email: reports@adonis.osti.gov

This report is available to the general public from:

National Technical Information Service

U.S. Department of Commerce

5285 Port Royal Road

Springfield, VA 22161

Telephone: $1-800-553-6847$ or

(703) $605-6000$

Fax: (703) 321-8547

Internet: http://www.ntis.gov/ordering.htm 


\title{
MHD-Induced Energetic lon Loss during H-mode Discharges in the National Spherical Torus Experiment (NSTX)
}

\author{
S. S. Medley, N. N. Gorelenkov, R. Andre, R. E. Bell, D. S. Darrow, E. D. Fredrickson, \\ S. M. Kaye, B. P. LeBlanc, A. L. Roquemore and the NSTX Team \\ Princeton Plasma Physics Laboratory, Princeton, New Jersey, 08543, USA
}

\begin{abstract}
Email: medley@pppl.gov
\end{abstract}

\section{Abstract}

MHD-induced energetic ion loss in neutral beam heated $\mathrm{H}$-mode discharges in NSTX is discussed. A rich variety of energetic ion behavior resulting from magnetohydrodynamic (MHD) activity is observed in NSTX using a horizontally scanning neutral particle analyzer whose sightline views across the three co-injected neutral beams. Reconnection events, sawteeth and bounce fishbones are observed to cause prompt loss (or redistribution) of energetic ions $(E \sim 10-100 \mathrm{keV})$ on a time scale of $\leq 1 \mathrm{~ms}$ and, consequently, a precipitous drop in the neutron yield. On the other hand, onset of low-n MHD modes leads to a much slower decay of the energetic ions and neutron yield. MHD-induced ion loss during $\mathrm{H}$-mode operation in NSTX exhibits yet another phenomenon. After H-mode onset, the NPA spectrum usually exhibits a significant loss of energetic ions mainly for E > $E_{b} / 2$ where $E_{b}$ is the beam injection energy, although this loss occasionally extends to lower energy. The magnitude of the energetic ion loss diminishes with increasing tangency radius of the NPA sightline, increasing toroidal field and increasing NB injection energy. Modeling suggests that MHD-induced ion loss is enhanced during $\mathrm{H}$-mode operation due to an evolution of the $q$ and beam deposition profiles that feeds both passing and trapped ions into the region of the plasma affected by the low-n MHD activity. Analysis of the particle interaction with a model magnetic perturbation supports the energy selectivity of the observed MHD-induced loss. Transport analysis using a fast ion diffusion model to emulate MHD-induced energetic ion loss shows significant modifications of the heating produced by the neutral beam which changes the inferred power balance, thermal 
diffusivities and the thermal energy confinement time. An accounting of energetic ion loss is therefore important for proper analysis of power balance and transport in plasmas exhibiting MHD-induced energetic ion loss.

\section{Introduction}

The National Spherical Torus Experiment (NSTX) [1] is a midsize low aspect ratio fusion research facility with auxiliary heating from Neutral Beam Injection (NBI) and High Harmonic Fast Wave (HHFW) launch. Typical NSTX parameters are major radius $R_{0}=$ $0.85-0.9 \mathrm{~m}$, minor radius $a=0.67 \mathrm{~m}$ resulting in an aspect ratio of $A=R_{d} / a \sim 1.3$, plasma current $I_{\mathrm{p}}=0.3-1.5 \mathrm{MA}$ and toroidal field $B_{\mathrm{T}}=0.3-0.6 \mathrm{~T}$. Three co-directed deuterium neutral beam sources have injected power up to $P_{\mathrm{NB}}=7 \mathrm{MW}$ at neutral energies up to $E_{\mathrm{b}}$ $=100 \mathrm{keV}$. HHFW heating at $30 \mathrm{MHz}$ has delivered up to $P_{\mathrm{RF}} \sim 5 \mathrm{MW}$ to deuterium and helium plasmas.

Determining the attractiveness of the spherical torus concept [2] in the areas of high- $\square$ stability, confinement, non-inductive current drive and divertor physics for pulse lengths much longer than the current relaxation time is the mission of the NSTX device. The performance achieved to date in NSTX has been reported elsewhere [3-6]. H-modes triggered by NBI heating are routinely obtained in NSTX and have become a standard operational scenario [7]. L-H transitions triggered by NBI heating have been obtained over a wide parameter range in $\mathrm{I}_{\mathrm{p}}, \mathrm{B}_{\mathrm{T}}$ and $\mathrm{n}_{\mathrm{e}}$ in either lower-single-null or double-null diverted discharges with elongation $\square$ up to 2.4 , triangularity $\square$ up to 0.8 and plasma pulse length approaching $1 \mathrm{~s}$. To date NSTX has achieved, non-simultaneously, stored energies up to $0.39 \mathrm{MJ}$, energy confinement times $\square_{\mathrm{E}}<0.12 \mathrm{~s}$ and $\mathrm{Q}_{\mathrm{T}} \leq 35 \%[3] ; \mathrm{T}_{\mathrm{T}}=\langle\mathrm{p}\rangle /\left(\mathrm{B}_{0}{ }^{2} / 2 \square_{0}\right)$ where $\langle p\rangle$ is the volume averaged total pressure and $B_{0}$ is the vacuum magnetic field at $\mathrm{R}_{0}$.

The magnetic field topology in spherical tokamaks can cause energetic ion behavior to differ in several aspects, sometimes subtle, from that in conventional aspect ratio tokamaks. To begin with, spherical tokamaks operate at significantly lower magnetic field than do most tokamaks; e.g. $\mathrm{B}_{\mathrm{T}}=0.3-0.6 \mathrm{~T}$ in NSTX compared to $2-5 \mathrm{~T}$ in 
conventional aspect ratio tokamaks. In conventional tokamaks, the poloidal magnetic field strength typically is much less than the toroidal field. As a result, the radial excursion of an energetic ion drift orbit is roughly the size of a poloidal gyroradius, which is considerably greater than the size of the Larmor radius. In spherical tokamaks, however, the poloidal magnetic field strength can exceed that of the toroidal field and the radial extent of the drift orbit can be comparable to the Larmor orbit. In conjunction with a relatively small major radius relative to the minor radius, this leads to modification of orbit topologies $[2,8,9]$, particle drifts [10], behavior of Alfvén-type instabilities [11,12], and neoclassical transport $[13,14]$. For example, the gyroradius of $80 \mathrm{keV}$ D co-injected NB heating ions in NSTX can be $\sim 0.3 \mathrm{~m}$ at the outboard midplane of the plasma. This is a sizable fraction of the $0.68 \mathrm{~m}$ minor radius of a typical plasma. As a result, energetic ion orbits in NSTX exhibit some features not seen in conventional tokomak orbits. For instance, banana orbits have larger radial excursion near the banana tip than near their midplane crossings, which is the widest part of the orbit in a standard tokamak. New orbit types can exist [2,8,9]; for example, those with no mid-plane crossing (teardrop orbits) and those with magnetic mirrors at four places.

The large fast-ion gyroradius in a spherical tokamak also can be comparable to the gradient scale length of the magnetic field, meaning that the energetic ion magnetic moment $(\square)$ may not be well conserved and energetic neutral beam ions could be nonadiabatic. The magnetic moment, $\square$, is the first order term of the magnetic moment polynomial expansion in terms of the adiabaticity parameter and is given by $\square=E_{\square} / B$, where $E_{\square}$ is the particle energy perpendicular to the magnetic field and $B$ is the magnetic field strength. If $\square$ invariance is broken, then it is possible that orbit stochasticity can cause fast ions to be lost. Indeed, stochasticity in NB ion orbits in NSTX has been found in orbit modeling [15] due to resonant interaction between gyromotion and bounce motion. However, due to the conservation of the canonical angular momentum, the net effect of the loss of $\square$ invariance is quite small and changes in magnetic moment are not expected to lead to a significant reduction in the confinement of co-injected beam ions in NSTX [16].

Prompt loss to the walls can also be augmented by the large gyroradius of energetic ions in spherical tokamaks as evidenced by numerical simulations [17]. The prompt loss of 
neutral beam ions from NSTX is expected to be between $12 \%$ and $42 \%$ of the total beam power, depending on the plasma current (1 MA to 0.6 MA). Such losses are diagnosed either from temperature measurements of ion deposition on first wall structures or by Faraday cup probes [18] which detect ions on loss orbits. Losses of ions with energy above $1 \mathrm{keV}$ in NSTX have been measured with a Faraday cup probe located at the vessel midplane [19]. In addition to this prompt loss to first-wall structures, orbit excursions beyond the separatrix combined with high atomic and molecular density enveloping the plasma can result in a depletion of the energetic ion population due to the energydependent charge-exchange losses. This can create a 'bump-on-tail' in the energetic ion distribution [20] that could excite instabilities and lead to further loss processes.

Resonant interaction of high-energy particles with magnetic perturbations in toroidal devices can produce large-scale modification of the particle distribution, sometimes leading to particle loss. Spherical tokamaks, including NSTX [21,22], START [23] and MAST $[24,25]$, are particularly susceptible to fast-ion driven instabilities, due primarily to their relatively low toroidal field, but also due to the direct effect of the low aspect ratio. The Alfvénic modes are readily excited in NSTX as the neutral-beam full-energy ion velocity is typically two to four times the Alfvén speed and because the Larmor radius of the fast ions is large compared to the minor radius, enhancing mode-particle interactions. Several classes of energetic-ion-induced MHD modes have been observed in NSTX and clear evidence of energetic ion losses has been seen in conjunction with at least two forms these modes [22]. For most NSTX beam heated plasmas, toroidal Alfvén eigenmodes (TAE) are quasi-continuous and have no detectable effect on plasma performance. However, in experiments in which $\mathrm{H}$-modes with broad density profiles were created in plasmas with relatively high q on axis, the TAE became strongly bursting and chirped down in frequency. Concurrently with the bursts, rapid drops $(10 \%-15 \%)$ in the neutron rate were observed as well as bursts of energetic-ion loss and $D_{a}$ line emission. These modes differ substantially from the usual precession-resonance fishbone mode seen in conventional aspect ratio tokamaks. They have been identified as bounce precession frequency fishbone modes [26] that are predicted to be important in high current, low shear discharges with a significant population of trapped particles having a large mean 
bounce angle, such as produced by near tangential beam injection into a small aspect ratio device like NSTX [26].

This paper addresses low-n MHD-induced energetic ion loss, specifically in $\mathrm{H}$-mode discharges where the broad, high-density profiles play a significant role in the loss process. For reference purposes, observations of classical ion behavior in MHD quiescent plasmas are presented in Section 2. The phenomenology of MHD-induced energetic ion loss observations in $\mathrm{H}$-mode discharges is presented in Section 3. In Section 4, TRANSP modeling is used to develop a plausible mechanism that accounts for the enhancement of MHD-induced ion loss in H-mode plasmas. Use of the ORBIT code to account for the observed energy dependence of the ion loss is discussed in Section 5. An analysis of the impact of energetic ion loss on TRANSP calculations of the neutron yield, power balance and transport is given in Section 6. The penultimate section gives a discussion of the reported experimental observations and numerical simulations and a summary is given in Section 8.

\section{Classical Behavior in Quiescent Plasmas}

Before describing the effects of MHD activity, it is useful to establish a baseline for energetic ion behavior in quiescent plasmas in NSTX derived from NPA measurements. First a description of the diagnostic used to measure energetic ion distributions is given. The NPA diagnostic on NSTX utilizes a PPPL-designed EIIB spectrometer [27] which measures the energy spectra of $\mathrm{H}$ and $\mathrm{D}$ neutrals simultaneously with a time resolution of $\sim 1 \mathrm{~ms}$ set by signal-to-noise levels. The calibrated energy range is $\mathrm{E}=0.5-150 \mathrm{keV}$ and the energy resolution varies from $\square E / E=7 \%$ at low $E$ to $\square E / E=3 \%$ at high $E$. The detector consists of a large area microchannel plate that is provided with two rectangular, semicontinuous active area rows for detecting $H$ and $D$. Each mass row has 39 energy channels. The NPA measures Maxwellian spectra of residual hydrogen in deuterium plasmas to obtain ion temperatures [28] and deuterium energetic ion spectra produced by injection of neutral beams into deuterium plasma [29]. The NPA views across the coinjection paths of the three neutral beam lines on NSTX that inject at $R_{\mathrm{NB}}$ of $\sim 0.7 \mathrm{~m}$ 
(source A), $0.6 \mathrm{~m}$ (source B) and $\sim 0.5 \mathrm{~m}$ (source $\mathrm{C}$ ) as shown in Figure 1. In NSTX, by convention the neutral beam tangency radii are positive for injection in the co-direction. On the other hand, the convention adopted for the NPA is that positive sightline tangency radii correspond to viewing co-directed ions.

The horizontal scanning capability for the NPA over a sightline tangency range of $R_{\text {tan }}$ $=1.25 \mathrm{~m}$ to $R_{\mathrm{tan}}=-0.75 \mathrm{~m}$ has enabled measurement of the anisotropic energy distribution of the beam ions, as illustrated in Figures $2 a$ and $2 b$. These spectra were derived from energy distributions measured on a shot-to-shot basis over eleven semi-reproducible discharges. For these L-mode discharges, $I_{\mathrm{p}}=0.85 \mathrm{MA}, B_{\mathrm{T}}=0.58 \mathrm{~T}$ and source $\mathrm{B}$ was injected with $E_{\mathrm{b}}=80 \mathrm{keV}, P_{\mathrm{NB}}=1.7 \mathrm{MW}$ in the time interval $\mathrm{t}=100-200 \mathrm{~ms}$ during the current ramp up. Over this time the line average electron density varied from $n_{\mathrm{e}}=1.2-$ $2.0 \times 10^{19} \mathrm{~m}^{-3}$. Variation of the electron temperature from Multi-Pulse Thomson Scattering (MPTS) [30] was $T_{\mathrm{e}}(0)=0.4-0.7 \mathrm{keV}$ and the ion temperature from Charge Exchange Recombination Spectroscopy (CHERS) [31] was $T_{i}(0)=0.3-1.0 \mathrm{keV}$. Details about these and other NSTX diagnostics can be found elsewhere [32]. Figure 2a shows the spectrum obtained $10 \mathrm{~ms}$ after start of injection. Here the spectrum peaks around the beam injection tangency radius, $R_{\mathrm{NB}} \sim 0.6 \mathrm{~m}$, and the full, half and third beam energy components are evident. The spectrum is highly anisotropic with the ion population depleting with decreasing NPA tangency radius. This occurs because decreasing tangency radius corresponds to decreasing pitch and therefore to beam deposition on trapped orbits, whereas the co-injected tangential NB deposits ions primarily on passing orbits with pitch approaching unity. Figure $2 \mathrm{~b}$ shows the spectrum measured $40 \mathrm{~ms}$ later at which time the beam ions have slowed down substantially so that the beam fractional energies are barely evident. Evidence of the spectrum filling in below the critical energy $E_{\text {crit }} \sim 15 \mathrm{keV}$ due to pitch angle scattering can be seen.

It is instructive to compare the measurements with the classical characteristic slowing down and $90^{\circ}$ pitch angle scattering times based on Coulomb collision theory [33]. For the present purposes, a deuterium beam ion with mass $A_{b}$, charge $Z_{b}$, and full energy $E_{b}$ is considered. As introduced by Stix [34], the time for such an energetic ion to thermalize is given by 


$$
\square_{t h}=\frac{\square_{s e}}{3} \ln \left[1+\left(E_{b} / E_{c r i t}\right)^{3 / 2}\right]
$$

where $\bigsqcup_{e}$ is the slowing-down time on electrons first defined by Spitzer [35]:

$$
\square_{s e}=6.27 \square 10^{14} \frac{A_{b} T_{e}^{3 / 2}}{Z_{b}^{2} n_{e} \ln \square_{e}} .
$$

$E_{\text {crit }}$, the critical energy at which ions and electrons receive a equal energy transfer from the beam ions, is given by

$$
E_{\text {crit }}=14.8 A_{b} T_{e}\left(\frac{\left[Z_{i}\right]}{A_{i}}\right)^{2 / 3}
$$

where the average charge to mass ratio of the bulk ions (denoted by the subscript ' $i$ ') is

$$
\frac{\left[Z_{i}\right]}{A_{i}}=\frac{\square_{j} n_{j}\left(Z_{j}^{2} / A_{j}\right) \ln \square_{j}}{n_{e} \ln \square_{e}}
$$

and the subscript ' $j$ ' indexes the bulk and impurity ion species. The time for $90^{\circ}$ pitch angle scattering [36,37], $\square_{\text {scat }}$, for full energy deuterium ions can be expressed as

$$
\square_{\text {scat }}=1.11 \square 10^{13} \frac{A_{b}^{1 / 2} E_{b}^{3 / 2}}{Z_{b}^{2} Z_{e f f} n_{e} \ln \square}
$$

where

$$
Z_{\text {eff }}=\frac{1}{n_{e} \ln \square} \square n_{j} Z_{j}^{2} \ln \square_{j}
$$


and the subscript ' $j$ ' indexes the bulk and impurity ion species. Units in the above expressions are time(s), energy $(e V)$, mass $(A M U), T_{e}(e V)$ and $n_{e}\left(m^{-3}\right)$.

For the discharges in which the spectra shown in Figures $2 a$ and $2 b$ were measured, parameters characteristic of the plasma core are $\mathrm{T}_{\mathrm{e}}(0) \sim 1000 \mathrm{eV}, \mathrm{n}_{\mathrm{e}}(0) \sim 5 \times 10^{19} \mathrm{~m}^{-3}, \mathrm{Z}_{\text {eff }} \sim$ 2, $\ln \square \sim 15,\left[Z_{\mathrm{i}}\right] / \mathrm{A}_{\mathrm{i}} \sim 1.03$ and, $\mathrm{E}_{\text {crit }} \sim 15 \mathrm{keV}$ yielding a slowing down time of $\mathrm{C}_{\mathrm{e}} \sim 52 \mathrm{~ms}$ and a thermalization time of $\square_{h} \sim 46 \mathrm{~ms}$ for $80 \mathrm{keV}$ deuterium beam ions. On the other hand, the time for $90^{\circ}$ pitch angle scattering for full energy deuterium ions under these conditions is $\square_{\text {cat }} \sim 236 \mathrm{~ms}$, about five times the thermalization time.

In these discharges, measurement of the pitch angle scattering is limited by the short duration the beam pulse and evolution of electron density and temperature. Nevertheless, these measurements indicate that the thermalization $\left(\square_{\mathrm{h}} \sim 50 \mathrm{~ms}\right.$ ) and pitch angle scattering ( $\square_{\text {ccat }}>100 \mathrm{~ms}$ ) times of energetic ions in NSTX quiescent plasmas are consistent with classical behavior.

Competition between ion energy diffusion and electron drag can give rise to a slope [38] of the ion energy distribution above the neutral beam injection energy of the form $f(E)$ $\sim \exp \left(-E / T_{\text {eff }}\right)$, where $T_{\text {eff }}$ given by

$$
T_{\text {eff }}=\frac{T_{i}+\left(E / E_{\text {crit }}\right)^{3 / 2} T_{e}}{1+\left(E / E_{c r i t}\right)^{3 / 2} \pm \square_{s e} 9.58 \times 10^{11} \frac{Z_{b}}{A_{b}} \frac{\left|E^{D^{*}}\right|}{\mathrm{v}_{\mathrm{b}}}\left(E / E_{c r i t}\right)^{3 / 2}}
$$

is an effective temperature that is a weighted average of the ion and electron temperatures. The last term in the denominator which accounts for the effect of the toroidal electric field, $\left|E^{*}\right|$, is $O \sim 10^{-2}$ in NSTX and can be neglected. Thus for $\mathrm{E}_{\text {crit }} \sim 15$ $\mathrm{keV}, \mathrm{E}=80 \mathrm{keV}$ and $\mathrm{T}_{\mathrm{i}} \sim 2 \mathrm{~T}_{\mathrm{e}} \sim 1 \mathrm{keV}$ typical of neutral beam heated discharges, $\mathrm{T}_{\text {eff }} \sim \mathrm{T}_{\mathrm{i}} / 2$ $\sim 0.5 \mathrm{keV}$. Hence, the slope of the neutral beam distribution above the injection energy is steep and, in fact, cannot be resolved with the NPA energy resolution mentioned earlier. Hence the absence of measurable slope in NSTX NB spectra such a shown in Figures 2a and $2 b$ is expected. 
Other experiments conducted on NSTX support classical behavior of energetic ions in discharges exhibiting benign MHD activity. The confinement of dilute populations of beam ions in NSTX was investigated by injecting short $~ 3 \mathrm{~ms}$ pulses (or "blips") of $80 \mathrm{keV}$ deuterium neutrals [39]. The neutron decay rate versus time after the beam blip ends was compared with the decay rate expected due to collisional slowing down as well as the evolution of the energetic ion spectra measured by the neutral particle analyzer. The temporal evolutions of the neutron yield and neutral particle spectra were found to be consistent with Coulomb scattering rates, implying an effective beam-ion confinement time $>100 \mathrm{~ms}$.

High Harmonic Fast Wave (HHFW) launch [40] has been employed on NSTX to provide both auxiliary electron heating [41] and current drive in Ohmic plasmas. The $\mathrm{HHFW}$ frequency is $30 \mathrm{MHz}$ with a variable spectrum of wavenumbers $k_{/ /}=7-14 \mathrm{~m}^{-1}$ and power levels up to $P_{R F} \sim 5 \mathrm{MW}$. When HHFW and NBI heating are combined, acceleration of energetic ions above the NB full energy is observed. For a typical deuterium NB injection energy of $80 \mathrm{keV}$, the energetic ion 'tails' can extend up to $130 \mathrm{keV}$ and enhancement of the neutron yield is also observed. Following turn off of the HHFW power, both the RF-induced ion tail and the elevated neutron yield decay to NB only values on a time scale consistent with classical slowing down [42].

\section{Energetic Ion Loss Observations}

A rich variety of energetic deuterium ion behavior resulting from magnetohydrodynamic (MHD) activity has been observed in NSTX [43] using the neutral particle analyzer diagnostic. The appearance of MHD activity can have a pronounced effect on both the 'thermal' ( $E \sim 0.5-10 \mathrm{keV}$ ) and 'energetic' ( $E \sim 10-100 \mathrm{keV}$ ) ion populations in NSTX. For example, onset of an $n=1$ or $n=2$ mode often leads to rollover

and relatively slow decay of the energetic ion population and, consequently, the neutron yield. The effect of reconnection events [29], sawteeth and bounce fishbones [26] differs from that observed for low-n MHD modes. In these cases, prompt loss of the energetic ion 
population occurs on a time scale of $\leq 1 \mathrm{~ms}$ and a precipitous drop in the neutron yield occurs.

This section deals with low-n MHD-induced energetic ion loss, specifically in $\mathrm{H}$ mode discharges where broad and high-density profiles play a significant role in the loss process. The analysis presented herein draws on a database of NSTX discharges run in the late 2002 and early 2003 time period. A subset of the most relevant discharges is given in Table 1. Here the percentage decrease (loss) of the NPA signal at $E=60 \mathrm{keV}$ was measured at an interval of time $\square \mathrm{t}_{\text {Loss }}(\mathrm{s})$ after the $\mathrm{H}$-mode onset time. The discharges were all double null diverted with $I_{p}=0.8 \mathrm{MA}$ (except as noted) and deuterium neutral beam injection energies spanning $80-100 \mathrm{keV}$. H-mode transitions occurred near the start of the current flattop except for a late transition case in SN109828. SN108730 was selected for detailed discussion here and additional performance features of this discharge were reported previously [5].

Table 1 MHD-Induced Energetic lon Loss Database

\begin{tabular}{|c|c|c|c|c|c|c|}
\hline $\begin{array}{c}\text { Shot } \\
\text { Number }\end{array}$ & $\begin{array}{c}\text { NPA } \\
R_{\text {TAN }}(\mathrm{cm})\end{array}$ & $B_{T}(T)$ & $\begin{array}{c}E_{b 0}(k e V) \\
A / B / C\end{array}$ & $\begin{array}{l}\text { Loss (\%) } \\
@ 60 \mathrm{KeV}\end{array}$ & $\begin{array}{l}\text { H-mode } \\
\text { Onset (s) }\end{array}$ & $\bar{\nabla} t_{\text {Loss }}(\mathbf{s})$ \\
\hline 108720 & 92 & 0.44 & $90 / 90 / 0$ & 71 & 0.226 & 0.209 \\
\hline 108728 & 92 & 0.53 & $90 / 90 / 0$ & 50 & 0.240 & 0.080 \\
\hline 108729 & 92 & 0.48 & $90 / 90 / 0$ & 68 & 0.245 & 0.150 \\
\hline 108730 & 70 & 0.49 & $90 / 90 / 0$ & 75 & 0.230 & 0.205 \\
\hline 108731 & 45 & 0.49 & $90 / 90 / 0$ & 86 & 0.245 & 0.240 \\
\hline $108872^{*}$ & 90 & 0.44 & $100 / 92 / 0$ & 50 & 0.258 & 0.180 \\
\hline 109015 & 70 & 0.40 & $80 / 80 / 0$ & 86 & 0.228 & 0.156 \\
\hline 109070 & 92 & 0.49 & $95 / 95 / 80$ & 56 & 0.228 & 0.129 \\
\hline $109828^{*}$ & 48 & 0.44 & $80 / 80 / 0$ & 72 & 0.358 & 0.077 \\
\hline 110184 & 70 & 0.44 & $80 / 80 / 0$ & 90 & 0.182 & 0.356 \\
\hline
\end{tabular}


An example of MHD-induced energetic ion loss during an H-mode for NSTX discharge SN108730 is presented in Figure 3. The upper panel shows the NPA spectrum of energetic NB deuterium ions as a function of energy and time. Following $\mathrm{H}$-mode onset at $230 \mathrm{~ms}$, the spectrum exhibits a significant loss of energetic ions only for $E>E_{b} / 2$ (encircled region). Selected discharge waveforms are shown in the lower panel. From the top, this is an $I_{\mathrm{p}}=0.8 \mathrm{MA}$ discharge with sources $\mathrm{A}$ and $\mathrm{B}$ injecting a total of $P_{\mathrm{NB}}=4.2 \mathrm{MW}$ of power with an injection energy of $E_{b}=90 \mathrm{keV}$. At the onset of the $\mathrm{H}$-mode marked by the dashed line passing through the drop in the $D_{\square}$ signal, little change is observed in the evolution of the neutron yield, $S_{n}$, the toroidal rotation velocity from the CHERS diagnostic, $\mathrm{V}_{\square}$, or the NPA signal, $\mathrm{S}_{\mathrm{NPA}}$, shown here for $60 \mathrm{keV}$ deuterium neutrals just above the beam half energy $E_{b} / 2=45 \mathrm{keV}$. Shortly afterwards around $280 \mathrm{~ms}$, however, the neutron yield and toroidal rotation velocity clamp concurrently with onset of decay in the NPA signal. The last panel shows the Mirnov spectrogram identifying the MHD mode activity. Although $\mathrm{n}=1$ activity prior to $\mathrm{H}$-mode onset arguably might affect the NPA signal evolution, this period is complicated by NB turn on events and will not be examined further. In the first $\sim 50 \mathrm{~ms}$ of the H-mode, MHD activity vanishes to give a quiescent phase where $S_{N P A}$ remains relatively constant. At $\sim 280 \mathrm{~ms}$, strong $n=3,2$ activity arises with mode amplitude $\square B \sim 0.5$ Gauss and simultaneously $S_{N P A}$ begins to decay and the neutron yield clamps. In the time interval from $280-400 \mathrm{~ms}, \mathrm{~S}_{\mathrm{NPA}}$ diminishes by $\sim 75 \%$. Depletion of energetic ions with $E>E_{b} / 2$ 'saturates' and persists until termination of the $\mathrm{H}$ mode by a reconnection event.

Figure 4 shows MPTS measurements of the electron density profiles around the time of $\mathrm{H}$-mode onset (upper panel) and evolution of the electron temperature (diamonds) and line integral density (circles) overlaid with waveforms for plasma current and beam power (lower panel). The electron density profiles correspond to times flagged by colorcoded solid circles on the trace for the line integral density. In the electron density profile plot, the shaded region denotes the approximate radial location of the source of the NPA signal. As discussed below, TRANSP simulation shows that the NPA charge exchange signal arises primarily in the region where the NPA sightline and NB injection axes 
intersect. This is because the density of beam primary and halo neutrals in this region exceeds the passive wall neutral density by at least an order of magnitude.

The TRANSP code $[44,45]$ is capable of simulating the NPA neutral flux measurements including horizontal and vertical scanning of the sightline. In the top panel of Figure 5 it can be seen that prior to $\mathrm{H}$-mode onset (upper spectra) reasonable agreement exists between measurement and simulation at all energies. The observed MHD-induced ion loss during the $\mathrm{H}$-mode (shaded region in the lower spectra) is not reproduced by TRANSP, of course, since the code does not contain a model for this process but otherwise the two spectra also agree reasonably well. From the TRANSP simulation one may infer that plasma opacity effects due broad electron density profiles characteristic of $\mathrm{H}$-modes neither impair beam deposition (e g. by depositing beam ions on prompt loss orbits) nor cause the observed ion loss.

The neutron production in NSTX is predominantly from beam-target reactions. Thus the neutron rate is a robust measure of the energetic ion population. As seen in the lower panel of Figure 5, for SN108730 the neutron yield calculated by TRANSP exceeds the measured neutron yield following MHD onset during the H-mode phase by $\sim 25 \%$. This neutron rate discrepancy will be further addressed in Section 6.

The magnitude of the energetic ion loss (decrease of $S_{N P A}$ in the region $E>E_{b} / 2$ ) was observed to diminish with increasing tangency radius, $R_{\text {tan }}$, of the NPA sightline and with increasing NB injection energy, $E_{b}$, as illustrated in Figure 6 . In addition, the loss diminishes with increasing toroidal field but a sparse data set exists since the available range was only $B_{\mathrm{T}}=0.44-0.53 \mathrm{~T}$.

Spatial localization of the NPA flux arises from the intersection of the diagnostic sightline with the NB sources as illustrated in Figures 7 and 8 . Figure 7 shows TRANSP calculation of the charge exchange emissivity for a $60 \mathrm{keV}$ deuterium energetic ion energy and a sightline tangency radius of $R_{\text {tan }}=0.7 \mathrm{~m}$ (blue curve) overlaid with the total beam neutral density for Source A (red curve). The curves are plotted as a function of distance along the NPA sightline measured from the pivot point of the diagnostic (see Figure 1). Also shown is the pitch angle variation along the sightline (black curve). As can be seen, the charge exchange emissivity is significantly localized due to charge exchange on the 
beam primary and halo neutrals. In this case, approximately $2 / 3$ of the integrated flux originates from the intersection of the NPA sightline with the NB region where ions are born on passing orbits. The remainder originates in the outboard regions of the plasma where ions are more likely to be deposited on trapped orbits. Note that the spatial localization of the NPA signal weakens with decreasing NPA tangency radius, $R_{\tan }$, due to attenuation of the beam neutral density with increasing penetration distance. The pitch angle 'range' sampled by the NPA is obtained from this plot by noting the values of the pitch angle at the rising and falling edges of the beam neutral density curve. This process was repeated for Sources $B$ and $C$ and for other NPA tangency radii to obtain the curves shown in Figure 8. Two features are to be noted. First, the pitch angle sampled by the NPA varies only weakly with the NB source. Second, the pitch angle range corresponding to the variation of ion loss with NPA $R_{\text {tan }}$ shown in Figure 6 is approximately $v_{\|} / v \sim 0.6-$ 1.0. Thus the NPA detects primarily passing energetic ions.

\section{TRANSP Analysis of the Energetic Ion Loss Mechanism}

TRANSP modeling suggests a possible mechanism causing the enhancement of MHD-induced ion loss observed during $\mathrm{H}$-mode operation as illustrated in Figure 9. Shown are TRANSP outputs for $\mathrm{t}=220 \mathrm{~ms}$ prior to the $\mathrm{H}$-mode (dashed blue curves) and $\mathrm{t}=\mathbf{2 8 0}$ ms during the $\mathrm{H}$-mode (solid red curves) corresponding to onset of the NPA signal decay (see Figure 3). The shaded bar near r/a $\sim 0.6$ denotes the predominant region of MHD activity detected by the ultra soft x-ray diagnostic arrays. MPTS input profiles are shown for the electron density and temperature in panels (a) and (e), respectively. Following the transition to the $\mathrm{H}$-mode, the evolution of these profiles drives a broadening and edgegradient steepening of the pressure profile that in turn evolves the plasma current density profile as shown in panel (f), this change being largely due to bootstrap driven current. As a result, the q-profile evolves so as to introduce a $q=2.5$ surface in the shaded region where MHD activity was identified as noted earlier. (There is as yet no direct measurement of the q profile on NSTX; it is inferred via fitting of the magnetics data with the code EFIT $[46,47]$.)

Thus low-n MHD activity arises as shown in the Mirnov spectrogram in panel (e). 
Concurrently, the broad density profile shifts a significant fraction of core-weighted beam deposition to the MHD-active region as shown in panel (b). Volume integration of the beam deposition over the region $0.3<\mathrm{r} / \mathrm{a}<0.9$ and conversion to power gives $\mathrm{P}_{\text {dep }}=1.0$ $\mathrm{MW}$ at $\mathrm{t}=0.220 \mathrm{~s}$ and $\mathrm{P}_{\text {dep }}=1.5 \mathrm{MW}$ at $\mathrm{t}=0.280 \mathrm{~s}$. An increased fraction of this outshifted power, $\square \mathrm{P}_{\text {dep }}=0.5 \mathrm{MW}$, is deposited on trapped banana orbits as shown in panel (c). From this we infer that MHD-induced ion loss is enhanced during $\mathrm{H}$-mode operation due to a congruous evolution of the q profile and beam deposition profile which feeds beam ions into the region of low-n MHD activity in the region r/a $\sim 0.6$, leading to depletion of the NPA energetic ion spectrum shown in panel (d). The reason for selective loss of the more energetic ions will be discussed in Sec. 5.

The above behavior is typical for discharges like SN108730 in which an early H-mode occurs shortly after turn on of the second neutral beam source. The contention that low- $n$ MHD activity and the H-mode must coexist to create enhanced ion loss is clearly illustrated in SN109828. As can be seen in Table 1, this discharge has a late $\mathrm{H}$-mode onset compared with other shots in the energetic loss database. Figure 10 shows selected waveforms for this discharge. The top panel provides the plasma current (blue curve), neutral beam heating power (red) and $\mathrm{D}_{\square}$ traces with the $\mathrm{H}$-mode transition at $358 \mathrm{~ms}$ marked by the dashed line through the drop in the $D_{\square}$ signal. Waveforms for the neutron rate, $S_{n}$, and the neutral particle analyzer signal, $S_{n p a}$, at $E_{b}=60 \mathrm{keV}$ are presented in the center panel. The Mirnov coil spectrogram in the bottom panel shows the MHD low-n mode activity. It can be seen that the $n=1,2,3$ activity that sets in at 300 ms appears to have little or no effect on the neutron and NPA signals, though this claim might be obscured by the coincident turn on of the second neutral beam. However, at the H-mode transition a marked reduction in the NPA signal occurs followed by a drop off in the neutron rate. Thus is can be concluded that MHD activity alone does not induce significant ion loss but that MHD activity in conjunction with an $\mathrm{H}$-mode does.

It must be emphasized that MHD-induced energetic ion loss during $\mathrm{H}$-modes is not a consequence of any $\mathrm{H}$-mode characteristic other than the broad, high-density profiles that invariably occur. MHD-induced energetic ion loss has also been occasionally observed in L-mode discharges that have unusually high, broad electron density profiles. 


\section{ORBIT Simulation of Energetic Ion Loss}

The ORBIT code $[48,49]$ was used to model the effect of MHD modes on the confinement of beam ions in NSTX. The ORBIT code, which can handle highly noncircular geometries as well as nonaxisymmetric magnetic field perturbations, follows beam ion guiding center orbits and calculates the effect MHD modes have on the energetic ion distribution. For realistic simulations we employ an EFIT numerical equilibrium and model MHD activity based on the experimental observations. MHD activity was measured as being dominated by one $m=4$ poloidal harmonic at $n=2$. The vector of the perturbed magnetic field is described by the formula $\Pi \mathbf{B}=\Pi \Pi \Pi \mathbf{B}$, where the scalar function $\square$ is approximated in a simple manner consistent with the MHD mode by

$$
\square \sim(1 \square n q / m)\left(r / r_{s}\right)^{m} \sin (n \square \square m \square)
$$

if $\mathrm{r}<\mathrm{r}_{\mathrm{s}}$ and decreases rapidly with minor radius at $\mathrm{r}>\mathrm{r}_{\mathrm{s}}$ where $\mathrm{r}_{\mathrm{s}}$ is the minor radius (square root of the normalized toroidal magnetic flux) of the rational magnetic surface and $\square$ is the toroidal angle. Note that in the simulations, $\square$ is normalized at its maximum to the amplitude $\square_{0}$. The magnetic field amplitude is approximately $\square B / B \sim m \square_{0}$ if the shear is small. In the simulations, the plasma toroidal rotation is included in the form of the radial electric field potential $\square \mathrm{keV}]=\square 54.3 \square\left(1 \square 0.93 \square^{0.1}\right)$, which is a monotonic function changing from zero at the plasma center to $-3.8 \mathrm{keV}$ at the edge. This provides a strong rotation of $\square_{\square}=2.4 \times 10^{5} \mathrm{rad}^{-1} \mathrm{~s}^{-1}$ in the core. To illustrate the mechanism of passing particle interaction with the mode we write the resonance condition, which includes the local Doppler shift due to the $E \times B$ drift,

$$
\square \square \square_{E \square B} \square\left(k_{\|}+l / q R\right) v_{\|}=0 .
$$


Here $\square$ is the MHD mode frequency and in the limit of high aspect ratio one can approximate $k_{\|}=(m \square n q) / q R$ and $l= \pm 1$ due to the poloidal modulation of the ion drift velocity components. Note that in NSTX due to the large radial width of the beam ion drift orbit, which is comparable with the minor radius and on the order of the mode width, $|l|$ may be larger than unity [50]. The MHD activity has approximately constant (and low) frequency $\left|\square \square \square_{E \square B}\right|<<\left|v_{\|}\right| / q R$. Hence for the passing particles (representing the majority of particles measured by the NPA) the resonance in Equation (9) is possible if $\left|k_{\|}+l\right|<<1$. For example, for the $m=4, n=2$ mode and $l= \pm 1$ the local resonance is possible near the $q=$ 2.5 surface. Due to the orbit width, however, particles interact with perturbations even if they satisfy the resonance condition only on part of their trajectory, which leads to high values of $l$. Note that the resonance condition of Equation (9) is velocity dependent, so that the low energy particles should have less response from the mode due to the smaller orbit width and smaller $v_{\|}$term, which leads to narrower radial locations of possible resonances.

In running the ORBIT code, beam ions were launched in the phase space where the NPA signal peaks (see Figure 7). The pitch of the observed particles at the moment of charge exchange with the neutrals is approximately given by the dependence $\square \equiv v_{\|} / v=1.5125 \square 0.629 R_{c x}[m]$ where $R_{\text {cx }}$ is the distance along the NPA sightline. Two groups of particles were investigated: passing and trapped. When the passing particles are launched they are initially distributed isotropically within the pitch range $0.8<0<0.9$ with a flat distribution. Trapped particles are distributed within the window $0.55<0<0.7$. At the end of the run, particles are recorded within $a \pm 0.02$ pitch angle range from the NPA viewing sightline (note the results are only slightly sensitive to this number). Beam ions are started with single energies of $E_{\mathrm{b}}=80$ and $40 \mathrm{keV}$ and are allowed to slow down and scatter due to collisions for $10 \mathrm{~ms}$. Figure 11a shows the results of simulations for passing particles with the two values of injected energies and reveals, as expected, that highenergy particles are more strongly affected by the perturbations. The signal from the highenergy ions is reduced by as much as $60 \%$ from the unperturbed case. The $40 \mathrm{keV}$ energy beam ions are less affected with the signal falling within the statistical error, which was 
around $20 \%$ in this case. It is interesting to note from Figure $11 \mathrm{~b}$ the correlation of the expected NPA signal with the value of the safety factor, which was scaled by multiplying the whole $q$-profile with a scaling factor, so that the factor 1.0 corresponds to the experimentally analyzed plasma. As was argued above, this sensitivity is expected due the $k_{\|}$dependence on $q$. The ORBIT simulations indicated that the trapped particles are not affected by the perturbation.

Introduction of the electric field, as the simulations show, changes the nature of the mode particle resonance interactions because the $E x B$ drift induces a Doppler shift that is a function of particle position. This is different from the recent modeling [51] in which the electric field and mode rotation frequency were ignored. In Ref [51], MHD perturbation induced a physical space island, whereas in our case due to large orbit widths the interaction is much broader and forms many islands in the phase space. As the simulations show, high harmonics $|l|>1$ result in stochastic phase space diffusion of passing ions.

With the ORBIT code one can study the losses of beam ions. Here we specify the initial distribution of beam ions assuming tangential injection at $E_{\mathrm{b}}=80 \mathrm{keV}$ similar to the conditions used in the experiment. Without the perturbation, beam ions are lost promptly with a loss fraction of about $9 \%$. MHD activity enhances these losses by almost doubling them at $\square_{0}=3 \times 10^{-4}$, as shown in Figure 12. For this case, the mode amplitude of the MHD activity measured at the plasma edge is $\square B / B \sim 2 \times 10^{-4}$, which is much less than the value required in ORBIT ( $\left.\square B / B \sim m \square_{0} \sim 12 \times 10^{-4}\right)$ for quantitative agreement between the ORBIT modeling the experiment results from NPA measurements. This is expected because the edge amplitude is smaller due to the envelope of the MHD mode decaying strongly towards the plasma edge and even further in the vacuum region where the measurements are made. Further analysis of this matter is outside the scope of this paper.

\section{Impact of Energetic Ion Loss on Neutron Yield and Transport Analysis}

It is well documented that MHD activity of various types can have various effects on ion behavior in both conventional [52,53] and spherical [26] tokamaks and in particular 
MHD activity can induce energetic ion loss. To palliate the lack of a physics-based model in TRANSP for MHD-induced loss of energetic ions, an existing capability to modify the energetic ion diffusion in a manner that approximates MHD loss is invoked. Note that energetic ion diffusion is generally small in conventional tokamaks [52] and there is no evidence so far that this is not the case in spherical tokamaks as well. So the use of enhanced energetic ion diffusion to emulate the observed MHD ion loss must not be construed as implying that elevated energetic ion diffusion is a feature unique to spherical tokamaks.

In the TRANSP model, fast ion diffusion can be enhanced over a specified region of physical space in the discharge corresponding to localization of the MHD activity as well as over a specified ion energy range to accommodate the energy-selective loss observed in the NPA measurements. The time dependence of the fast ion diffusion can also be specified. Two criteria were employed for determining the fast ion diffusion parameters: the first was to obtain agreement between the measured neutron rate and that generated by the kinetically based TRANSP simulation, and the second was to simultaneously obtain agreement with the measured energetic ion distribution as shown in Figure 5. The energetic ion diffusion model effecting this goal is as follows: (1) in time, the diffusivity was ramped up from a small value at the start of beam injection to $\square_{i}^{\text {Fast }}=3.8 \mathrm{~m}^{2} / \mathrm{s}$ at $\mathrm{t}=300 \mathrm{~ms}$ and thereafter increased gradually to a value of $\square_{1}^{\text {Fast }}=5.0 \mathrm{~m}^{2} / \mathrm{s}$ at $\mathrm{t}=600 \mathrm{~ms}$; (2) in energy, the diffusivity multiplier was increased from zero at $E_{\mathrm{D}}=45 \mathrm{keV}$ to unity at $E_{\mathrm{D}}=50 \mathrm{keV}$ where it remained constant until $E_{\mathrm{D}}=75 \mathrm{keV}$ and was then decreased to zero at $E_{\mathrm{D}}=80$ $\mathrm{keV}$ and remained so up to the injection energy of $E_{\mathrm{D}}=90 \mathrm{keV}$; (3) in space, the diffusivity was constant across the plasma minor radius. The selected energy range corresponds to the 'well' in the NPA ion distribution shown in Figure 5. The ORBIT simulation of the MHDinduced loss that was described in the previous section also supports this choice of energy range. TRANSP results obtained by applying this fast ion diffusion model to SN108730 are shown in Figures 13 - 19.

The neutron rates are shown in Figure 13. Here the red curve is the measured neutron rate, the blue curve is the simulated rate without fast ion diffusion, and the black curve is with fast ion diffusion. In both simulations, $Z_{\text {eff }}=2.5$ over the minor radius and 
$\mathrm{D} /(\mathrm{H}+\mathrm{D})=0.9$, constant in time. The effect on the energetic ion distribution at $\mathrm{t}=400 \mathrm{~ms}$ (when the ion loss has nearly saturated as evident in Figure 3) is show in Figure 14. Here the black curve is the measured energetic ion distribution, the red curve is the simulated distribution without fast ion diffusion, and the blue curve is with fast ion diffusion. As can be seen, an excellent match exists simultaneously between the neutron yield and energetic ion distribution measurements and the TRANSP simulation with fast ion diffusion.

As shown in the upper panel of Figure 15, invoking fast ion diffusion diminishes the neutral beam power input to both the electrons (dashed curves) and ions (solid curves) by the order of $20 \%$. The NB fast ion orbit loss to the first wall structures (lower panel) increases by $\sim 0.7 \mathrm{MW}$ as a result of the imposed fast ion diffusion. In the next few figures, it will be seen that the diminished power input to ions and electrons causes TRANSP to reduce the appropriate diffusivities (hence increase the thermal confinement times) in order to match the measured ion and electron temperatures used as input to the calculation,

The impact of this ion loss on transport and other parameters of interest is shown in Figures $16-19$. The volume-integrated ion power balance at $t=400 \mathrm{~ms}$ is shown in Figure 16 without (blue curves) and with (red curves) fast ion diffusion. (Normalized radius, r/a, is square root of toroidal magnetic flux normalized to total flux.) In general, the volume integrated powers for ion conduction and ion heating are lower with fast ion diffusion than without, as expected. Complementary results for the electron power balance with and without fast ion diffusion are given in Figure 17. In the power balance plots, $E_{\text {heat }}$ is the total electron heating (NB plus Ohmic and ion-electron coupling) and likewise for the total ion heating, $I_{\text {heat }}$, except minus the ion-electron coupling. In both figures, the errors in the local kinetic measurements and their integrated effects over the volume become important towards the edge.

Figure 18 shows profiles of the flux surface average thermal diffusivities extracted from TRANSP power and momentum balance analysis plotted against normalized minor radius for the time of interest. The extreme core and edge regions are excluded because of fluctuations arising from uncertainties in the measured values and/or gradients. In these calculations, heating source and loss terms are evaluated, and the unaccounted for "balance" is assumed to be diffusive and assigned a local diffusivity. Including energetic 
ion loss reduces the electron and ion diffusivities. A neoclassical prediction of the ion diffusivity, $\square^{\mathrm{NC}}$, obtained from the NCLASS code [54] follows more or less the shape of the $\square$ profile and is unchanged with the inclusion of fast ion diffusion. This is due to the fact that $\square_{1}^{\mathrm{NC}}$ is computed using the neoclassical ion thermal flux from NCLASS and the measured local gradient and density, ignoring energetic ions. Without fast ion diffusion, one sees that $\square_{\mathrm{i}} \square \square_{i}^{\mathrm{NC}}$ in the core region, but that $\square_{\mathrm{i}}<\square_{\mathrm{i}}^{\mathrm{NC}}$ in the edge region. A similar trend is observed with fast ion diffusion, but including fast ion diffusion causes $\square_{\text {th }}$ to plummet drastically in the outer region to $\square_{i} \ll \square_{i}^{\text {NC }}$. The energetic ion diffusivity, $\square^{\text {ast }} \sim 4$ $\mathrm{m}^{2} / \mathrm{s}$ (green line), modestly exceeds $\square_{i}^{\mathrm{NC}}$ outside the core region and $\square^{\text {Fast }} \gg \nabla_{i}$. As can be seen in the lower panel of Figure 3 , the central toroidal rotation velocity, $v_{\square}(0) \sim 250 \mathrm{kms}^{-1}$, is substantial for SN108730. Inclusion of fast ion diffusion also reduces the inferred momentum diffusivity, $\square_{\square}$, drastically as TRANSP responds to maintaining the measured input toroidal rotation in the face of $\sim 20 \%$ reduction of the NB drive. As a result, the momentum confinement time increases from $\sim 80 \mathrm{~ms}$ without fast ion diffusion to $\sim 160 \mathrm{~ms}$ with fast ion diffusion.

In most NSTX discharges, the electron thermal confinement is large relative to the ion thermal confinement [55] with $\square_{\mathrm{e}} \gg \square_{i}^{\mathrm{NC}}$ over the resolved normalized minor radius. This does not appear to be the case here since $\square_{e} \sim \square_{1}^{N C}$. Inclusion of fast ion diffusion, in fact, modestly reduces $\square$.

Figure 19a shows the total energy confinement time,, , and Figure $19 \mathrm{~b}$ the toroidal beta, $\square_{T}$, with and without fast ion diffusion. Of note here is that the confinement time with fast ion diffusion (red curve) is larger than without fast ion diffusion (blue curve). This is understandable, since loss of neutral beam heating power as a result of MHD-induced energetic ion loss naturally requires improved energy confinement to realize the measured input values for $T_{e}$ and $T_{i}$. On the other hand, toroidal beta decreases with the inclusion of fast ion diffusion. This is due to the fact that fast ion diffusion reduces both the parallel and perpendicular energetic ion betas by $\sim 25 \%$ due to a reduction in the energetic ion pressure profile. (Note that non-thermal ions usually contribute $\sim 25-30 \%$ to the stored energy and toroidal beta in NSTX.) 
It is instructive to apply a simple analytical model to the measured ion distribution presented in Figure 5 to estimate the energetic ion loss time, $\square_{\text {oss. }}$. The steady state distribution function should satisfy $S t(f)-f / \square_{\text {oss }}+Q-S=0$ where $Q$ and $S$ are the source and sink of beam ions, respectively, and $\square_{\text {oss }}$ is their characteristic loss time which is introduced following Ref. [56]. Beam ions are thermalized from the velocity at injection until they form the background species at low velocity. During slowing down, the beam ion distribution function satisfies the equation

$$
\frac{1}{\square_{\text {slow }} \mathrm{v}^{2}} \frac{\partial}{\partial \mathrm{v}}\left(\mathrm{v}^{3}+\mathrm{v}_{*}^{3}\right) \mathrm{f}-\frac{\mathrm{f}}{\square_{\text {loss }}}=0
$$

where $\square_{e}$ is the slowing down time on electrons and $\mathrm{v}_{*}$ is the critical velocity. The solution to Equation (8) is the familiar slowing down distribution function $f \sim 1 /\left(v^{3}+v_{*}^{3}\right)$ if $\square_{\text {oss }} 1$ I . At finite $\square_{\text {oss }}$ we obtain

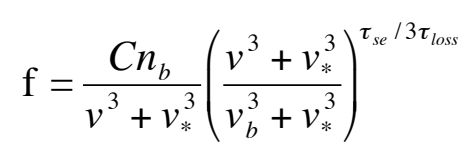

where $v_{b}$ is the beam ion birth velocity, $C$ is the normalization constant and $n_{b}$ is the beam ion density. By adjusting the loss time, an approximate fit of Equation (9) to the measured spectrum is obtained as shown in Figure 20 . This dependence translates to $\square_{\text {oss }}=\square_{b e} / 15$.

This approximate energetic ion loss time, $\square_{o s s}=\square_{s e} / 15$, can be compared with a loss time derived from the energetic ion diffusion model used in TRANSP. From TRANSP for SN108730, $\square_{\text {be }} \sim 60 \mathrm{~ms}$ in the plasma core region, so the 'analytic' calculation implies $\square_{\text {oss }} \sim$ 4 ms. Defining a 'numerical' loss time, $\square_{\text {Loss }}^{\text {RANSP }}$, as

$$
\square_{\text {Loss }}^{\text {RANSP }}=\frac{(\square L)^{2}}{\square_{i}^{\text {Fast }}}
$$


where $\square_{i}^{\text {Fast }}$ is the diffusivity from the fast ion diffusion model and $\Pi L$ is a characteristic diffusion distance. Taking $\square_{i}^{\text {Fast }}=4 \mathrm{~m}^{2} / \mathrm{s}$ and $\Pi L \sim 0.2 \mathrm{~m}$ (approximately the distance from the MHD active region to the plasma periphery demarked in Figure 9) yields a loss time, $\square_{\text {Loss }}^{\text {RANSP }} \sim 10 \mathrm{~ms}$ which agrees reasonably well with the analytic value. In the absence of MHD, the beam-ion confinement time in NSTX was determined to be $\geq 100 \mathrm{~ms}$ based on 'beam blip' experiments [39].

\section{Discussion}

For NSTX beam heated discharges, measurements show that $T_{e}<T_{i}[55]$ and progressive thermalization of NB injected fast particles into a thermal plasma maintains fairly constant central temperatures while the density increases continuously. Since the $\mathrm{NBI}$ system uses beam energies typically many times larger than the 1-2 keV electron temperature, one expects from classical slowing down physics of energetic particles that most $(\sim 2 / 3)$ of the neutral beam power would be deposited into the electron population, which is evidenced in the upper panel of Figure 15. The experimental observation that $\mathrm{T}_{\mathrm{e}}<$ $\mathrm{T}_{\mathrm{i}}$ during $\mathrm{NBI}$ despite the expected preferential electron heating suggests that the ion confinement is much better than that of the electrons. Another viewpoint has been to surmise that an anomalous process causes enhanced ion heating [57]. From the TRANSP transport analysis presented herein, it can be inferred that taking energetic ion losses into account partially ameliorates this recondite situation. This is because the more energetic ions are lost: i.e. $E_{b}>E / 2$ and it is this group that does most of the electron heating. Ions below the half energy provide only a modest amount of electron heating before they slow down below the critical energy and heat primarily thermal ions. Thus because of the MHDinduced energetic ion loss, electron heating is significantly less that one would expect.

In some TRANSP analyses, such as presented herein for SN108730, the calculated neutron rate significantly exceeds the neutron measurement as seen in Figure 13. The absolute error in the measured neutron rate is estimated to be $25 \%$ so the disparity exceeds the error bar shown in this figure. Since neutrons are produced primarily by beam-target reactions, it is not surprising that the fast ion diffusion had to be raised to a 
value that lead to the $\sim 20 \%$ loss of the energetic beam ions for $E>E_{b} / 2$ as seen in Figure 14. The fast ion diffusion used was $D \sim 4 \pm 1 \mathrm{~m}^{2} \mathrm{~s}^{-1}$ from $t=0.2-0.7 \mathrm{~s}$, which is significantly larger than the established 'quiescent plasma' energetic ion diffusion coefficient of $D<0.05 \mathrm{~m}^{2} \mathrm{~s}^{-1}$ reported for the Tokamak Fusion Test Reactor $[58,59]$ as well as a number of other conventional tokamaks [52]. Note that the reduction in power to the electrons is $\square \mathrm{P}_{\mathrm{e}} \sim 0.4 \mathrm{MW}$ but the power reduction to the ions is only $\square \mathrm{P}_{\mathrm{i}} \sim 0.2 \mathrm{MW}$. TRANSP compensates for the reduced power by reducing the electron and ion diffusivities shown in Figure 18 in order to obtain a match with the measured input values for $T_{e}$ and $T_{i}$. In the core region, $\square_{\square}<<\square_{\mathrm{e}} \square \square_{\mathrm{i}}^{\text {Fast }}<\square_{\mathrm{i}} \square_{1}^{\mathrm{NC}}$ with and without fast ion diffusion. In the midradius region, $\square_{\square}<\square<\square_{1}^{\mathrm{NC}} \sim \square_{\mathrm{e}} \square \square_{\mathrm{Fast}}^{\text {Fath }}$ with and without the fast ion diffusion model, though it can be seen that fast ion diffusion modestly reduces $\square_{\mathrm{e}}$ but markedly reduces $\square_{i}$. (This ordering of diffusivities is consistent with a previously reported transport analysis of a similar NSTX neutral beam heated H-mode discharge [55].) In turn, this leads to increased energy confinement time but to reduced toroidal beta as seen in Figure 19. As a result of accounting for MHD-induced energetic ion loss, energy confinement times for $\mathrm{H}$-mode discharges will be increased relative to L-mode discharges whereas the previously existing NSTX database shows that energy confinement times for $\mathrm{H}$-mode and the best L-mode discharges are usually comparable [55]. Note that in the referenced database the experimental energy confinement times are global (i. e. containing fast-ion stored energy without subtraction of energetic ion losses) and were determined from EFIT magnetic reconstruction of the NSTX plasma.

The results of ORBIT modeling offer an explanation for the observed reduction of ion loss with increasing NPA tangency radius, $R_{\text {tan }}$, beam injection energy, $E_{b}$, and toroidal field $B_{T}$ noted in Sec. 3. With regard to the beam energy scaling, higher energies drive more beam current resulting in increased peaking of the current profile in the core. As a result, the q-profile should be more monotonic causing the modes to be shifted closer to the core thus reducing the resonant ion loss. This $\mathrm{q}$ dependence can be seen in Figure $11 \mathrm{~b}$ where departure of the $q$ scaling factor from 1.0 reduces the passing particle loss for $E_{b}=80 \mathrm{keV}$. With regard to toroidal magnetic field scaling, increasing field modifies the mode structure of the magnetic perturbation as modeled by ORBIT. From Mirnov coil observations, larger 
$B$ reduces $\square B / B$ and decreases ion loss as seen in Figure 12. With regard to diminished loss with increasing NPA tangency radius, the NPA spatial localization at the NB intersection diminishes with increasing $R_{\text {tan }}$ and the observation region becomes out-shifted to include a greater fraction of trapped particles that are not affected by the MHD-induced ion loss according to the ORBIT modeling. Unfortunately, these plausible explanations of the MHD-induced energetic ion loss scaling cannot be adequately verified at this time because the evolution of discharge parameters was not sufficiently controlled in the available database shown in Table 1.

The ORBIT analysis with the modeled field perturbation indicated that neutral beam power loss due to magnetic perturbations is at most $\sim 15 \%$ whereas TRANSP analysis yields $\sim 20 \%$ in order to match the neutron rate and the measured NPA spectrum. The ORBIT analysis indicated that only passing particles are lost, although is it possible for trapped ions to be diffusively transported by low-n MHD activity [60]. Note that the fast ion diffusion model in TRANSP does not discriminate between passing and trapped ions. However, it may be possible that ORBIT somewhat underestimates the MHD-induced ion loss, since the magnetic perturbation used in the code is a simplified representation of the more complex experimentally observed MHD activity. Overall, however, agreement between the codes can be viewed as remarkably good especially given the very different modeling basis used in ORBIT and TRANSP.

These issues aside, it is clear that MHD-induced energetic ion loss needs to be taken into account in power balance and transport analysis. Without a physics-based model for this process, the only convenient mechanism to accomplish this in TRANSP is to force a match between the calculated and measured neutron rates. Another approach is to introduce a $20-40 \mathrm{~ms}$ 'notch' in NB injection during the MHD-induced ion loss phase. The fast ion diffusion model in TRANSP can then be tuned to match the measured neutron decay rate, thus experimentally establishing an appropriate value for the fast ion diffusivity. Such an approach would be contingent upon continuation of the H-mode and MHDinduced ion loss during the notch as well as minimal perturbation to other discharge parameters of importance. 
The MHD-induced energetic ion loss during H-modes generates a "bump-on-tail" beam ion distribution as seen in Figure 5. One can ask whether this bump-on-tail distribution drives high frequency Alfvén activity [20]. For the database given in Table 1, Mirnov spectrograms consistently show high frequency activity during $\mathrm{H}$-modes, but the scenario is confused by other semi-concurrent events such as turn on of an addition beam source. However, even for SN109828 shown in Figure 10 where H-mode onset occurs well after turn on of the second neutral beam there is no significant change in Mirnov activity during the $\mathrm{H}$-mode phase. Thus with the caveat of a limited database, it appears that the bump-on-tail energetic ion distribution generated by MHD-induced ion loss does not drive Alfvén activity in NSTX.

\section{Summary}

In quiescent or MHD benign NSTX discharges, NPA measurements of the energetic beam ion distribution are consistent with classical behavior. The appearance of strong MHD activity can have a variety of effects on both the 'thermal' ( $E \sim 0.5-10 \mathrm{keV})$ and 'energetic' ( $E \sim 10-100 \mathrm{keV})$ ion populations in NSTX. For example, onset of an $n=$ 1 or $n=2$ mode often leads to a rollover and relatively slow decay of the energetic ion population and, consequently, the neutron yield. The effect of reconnection events, sawteeth and bounce fishbones differs from that observed for MHD modes. In these cases, prompt loss of the energetic ion population occurs on a time scale of $\leq 1 \mathrm{~ms}$ along with a sharp drop in the neutron yield.

This paper focused on energetic ion loss during $\mathrm{H}$-mode operation in NSTX induced by low-n, low frequency MHD activity. After H-mode onset, the NPA charge exchange spectrum usually exhibits a significant loss of energetic ions only for $E>E_{b} / 2$ where $E_{b}$ is the beam injection energy, though in rare cases the loss was observed to extend down to $E_{b} / 3$. The magnitude of the energetic ion loss was observed to diminish with increasing values of the tangency radius, $R_{\tan }$, of the NPA sightline, toroidal field, $B_{T}$, and NB injection energy, $E_{b}$.

TRANSP modeling indicates that the effect is driven by the high, broad density 
profiles endemic to $\mathrm{H}$-modes. The postulated mechanism is that a pressure-driven evolution of the q profile introduces low-n MHD activity whilst the beam deposition profile broadens to feed passing and trapped ions into the MHD active region. It must be emphasized the MHD-induced ion loss mechanism is a pressure profile effect, which can sometimes occur in unusually high density L-mode discharges, but almost always is observed in $\mathrm{H}$-mode discharges because of the nature of the electron density profile.

ORBIT analysis supported the observed energy dependence of the ion loss and implied that the calculated $\sim 15 \%$ energetic ion loss involved only passing ions. A fast ion diffusion model in TRANSP utilized to emulate the MHD-induced energetic ion loss indicated that $\sim 20 \%$ loss of the neutral beam input power was needed in order to match the neutron rate and the measured NPA spectrum.

Accounting for MHD-induced energetic ion loss increases the plasma energy confinement time, but reduces the neutron yield and the toroidal beta. A proper accounting of energetic ion loss is therefore important for accurate analysis of power balance and transport in plasmas exhibiting MHD-induced energetic ion loss.

The observed scaling of this loss indicates that MHD-induced energetic ion loss can be ameliorated to a significant degree by increasing the neutral beam injection energy and/or the toroidal field. On the other hand, since MHD-induced energetic ion loss is seldom observed in L-mode discharges, NSTX could be operated in L-mode rather than

$\mathrm{H}$-mode. However, this is likely not an attractive option since $\mathrm{H}$-mode operation plays a significant role in extending many other NSTX performance parameters of interest.

\section{Acknowledgements}

This work was supported by the United States Department of Energy under contract number DE-AC02-76CH03073. We express gratitude to Tom Holoman and Doug LaBrie for excellent workmanship in installing and maintaining the NPA diagnostic and to Bob Kaita for discussions on classical ion behavior. 


\section{References}

[1] Ono M., et al., 2001 Nucl. Fusion 411435.

[2] Peng Y.-K. M. and Strickler D. J., 1986 Nucl. Fusion 26769.

[3] Menard J. E., et al., 2003 Nucl. Fusion 43330.

[4] Synakowski E.J., et al., 2003 Nucl. Fusion 431653.

[5] Gates D. A., et al., 2003 Phys. Plasmas 101659.

[6] Mangi R., et al., 2003 Plasma Phys. Control. Fusion 45657.

[7] Mangi R., et al., 2003 Nucl. Fusion 43969.

[8] Mikkelsen D. R., White R. B., Akers R. J., Kaye S. M., McCune D. C., and Menard J. E., 1997 Phys. Plasmas 43667.

[9] Grishanov N. I., de Azevedo C. A., and de Assis A. S., 1998 Phys. Plasmas 5705.

[10] Kolesnichenko Y. I., Marchenko V. S., and White R. B., 2001 Phys. Plasmas 8 3143.

[11] Gorelenkov N. N., et al., 2002 Nucl. Fusion 42977.

[12] Gorelenkov N. N., et al., 2003 Nucl. Fusion 43228.

[13] Gates D. A. and White R. B., "Collisional Transport in a Low Aspect Ratio Tokamak

- Beyond the Drift Kinetic Formalism," 2004 Princeton Plasma Physics Laboratory Report, PPPL-3917. (Submitted to Phys. Plasmas)

[14] Mynick H. E., White R. B., and Gates D. A., "Onmiclassical Diffusion in Low Aspect Ratios Tokamaks," 2004 Princeton Plasma Physics Laboratory Report, PPPL-3935. (Submitted to Phys. Plasmas)

[15] Yavorskij V. A., et al., 2002 Nucl. Fusion 421210.

[16] Egedal J., Redi M. H., Darrow D. S., and Kaye S. M., 2003 Phys. Plasmas 10, 2372

[17] Darrow D. S., et al., "Neutral Beam Ion Loss Modeling for NSTX," Controlled Fusion and Plasma Physics (Proc. of the $26^{\text {th }}$ EPS Conf., Maastricht, 1999), ECA Vol. 23J, European Physical Society, Geneva, (1999). CD-ROM file P4.090.

[18] Darrow D. S., Bell R., Johnson D. W., Kugel H., Wilson J. R., Cecil F. E., Maingi R., Krasilnikov A., and Alekseyev A., 2001 Rev. Sci. Instrum. 72, 784.

[19] Darrow D. S., et al., "Measurements of Prompt and MHD-Induce Fast lon Loss 
from National Spherical Torus Experiment Plasmas," IAEA-CN-94/EX/P2-01, Proc. 19 ${ }^{\text {th }}$ IAEA Fusion Energy Conference, Lyon, France, 14-19 October 2002. Also 2002 Princeton Plasma Physics Laboratory Report, PPPL-3754.

[20] McClements K. G., et al., 1999 Plasma Phys. Control. Fusion 41661.

[21] Gorelenkov N. N., et al., "Beam Ion Driven Instabilities in NSTX," 2004 Princeton Plasma Physics Laboratory Report, PPPL-3901. (In press)

[22] Fredrickson E. D., et al., 2003 Phys. Plasmas 102852.

[23] Akers R. J.,, et al., 2002 Nucl. Fusion 42122.

[24] Akers R. J., et al., 2002 Phys. Plasmas 93919.

[25] Akers R. J., et al., 2003 Plasma Phys. Control. Fusion 45 A175.

[26] Fredrickson E. D., Chen L., and White R. B., 2003 Nucl. Fusion 431258.

[27] Medley S. S. and Roquemore A. L., 1998 Rev. Sci. Instrum. 692651.

[28] Medley S. S., et al., 2003 Rev. Sci. Instrum. 741869.

[29] Medley S. S., Bell R. E., Darrow D. S., and Roquemore A. L., "Neutral Particle Analyzer Measurements of lon Behavior in NSTX," 2002 Princeton Plasma Physics Laboratory Report, PPPL-3668. (Also S. S. Medley, et al., Contributed Paper to $7^{\text {th }}$ IAEA TCM on Energetic Particles in Magnetic Confinement Systems, Göteborg, Sweden, (2001).

[30] LeBlanc B. P., Bell R. E., Johnson D. W., et al., 2003 Rev. Sci. Instrum. 741659.

[31] Bell R. E., et al., "Kinetic Profiles in NSTX Plasmas," 2001 Princeton Plasma Physics Laboratory Report, PPPL-3591.

[32] Johnson D. W., et al., 2003 Plasma Phys. Control. Fusion 451975.

[33] Sivukhin D. V., Reviews of Plasma Physics, Vol. 4, Consultants Bureau, New York (1966) 93.

[34] Stix T. H, 1972 Plasma Phys. 14367.

[35] Spitzer L. Jr., Physics of Fully lonized Gases, Interscience, New York (1962).

[36] Heidbrink W. W., 2002 Phys. Plasmas 928.

[37] Jassby D. L., 1977 Nucl. Fusion 17309.

[38] Fiore C. L., Medley S. S., Hammett G. W., Kaita R., Roquemore A. L., and Scott S. D., 1988 Nucl. Fusion 381315. 
[39] Heidbrink W. W., Miah M., Darrow D., LeBlanc B., Medley S. S., Roquemore A. L. and F. E. Cecil, 2003 Nucl. Fusion 43883.

[40] Ryan P. M., et al., 2001 Fusion Eng. Des. 56-57 569.

[41] Wilson J. R., et al., 2003 Phys. Plasmas 101733.

[42] Rosenberg A. L., et al., 2004 Phys. Plasmas. (In press)

[43] Medley S. S., Bell R. E., Fredrickson E. D., and Roquemore A. L., "Energetic Ion Behavior in the National Spherical Torus Experiment," 2003 Princeton Plasma Physics Laboratory Report, PPPL-3827.

[44] Budny R. V., et al., 1995 Nucl. Fusion 351497.

[45] Onega J., Evrard M., McCune D., 1998 Transactions of Fusion Technology 33182. [46] Lao L. L., et al., 1985 Nucl. Fusion 251611.

[47] Sabbagh S. A., et al., 2001 Nucl. Fusion 411601.

[48] White R. B. and Chance M. S., 1984 Phys. Fluids 272455.

[49] White R. B., 1990 Phys. Fluids B 2845.

[50] Putvinskij S. V., Reviews of Plasma Physics, Vol. 18 (B. B. Kadomtsev, Ed.), Consultants Bureau, New York (1986) 239.

[51] Carolipio E. M., Heidbrink W. W., Forest C. B., and White R. B., 2002 Nucl. Fusion 42853.

[52] Heidbrink W. W. and Sadler G. J., 1994 Nucl. Fusion 34535.

[53] Zweben S. J., Darrow D. S., Fredrickson E. D., Taylor G., von Goeler S., and White R.B., 1999 Nucl. Fusion 391097.

[54] Houlberg W. A., et al., 1997 Phys. Plasmas 43230.

[55] LeBlanc B. P., et al., "Confinement Studies of Auxiliary Heated NSTX Plasmas," 2003 Princeton Plasma Physics Laboratory Report, PPPL-3810. (Accepted by Nucl. Fusion)

[56] Cordey J. G., Goldston R. J., and Mikkelsen D. R., 1981 Nucl. Fusion 21581.

[57] Gates D. A., Gorelenkov N. N., and White R. B., 2001 Phys. Rev. Lett. 87 205003-1

[58] Ruskov E., Heidbrink W. W., and Budny R. V., 1995 Nucl. Fusion 351099.

[59] Heidbrink W. W., et al., 1991 Phys. Fluids B 33167.

[60] Mynick H. E.,1993 Phys. Fluids B 51471. 


\section{Figure Captions}

Figure 1. The Neutral Particle Analyzer (NPA) on NSTX views across the three neutral beam injection sources and can be scanned over a wide range of sightline tangency radii, $R_{\tan }$, on a shot-to-shot basis. $R_{\tan }$, is the perpendicular distance between the machine center and the NPA sightline.

Figure 2a. Shown is the NPA measurement of the NB ion distribution versus energy and NPA tangency radius near the start of neutral beam injection. The spectrum peaks around the beam injection tangency radius, $\mathrm{R}_{\mathrm{NB}} \sim 0.6 \mathrm{~m}$, and the full, half and third beam energy components are evident. The spectrum is highly anisotropic with the ion population depleting with decreasing NPA tangency radius, which corresponds to decreasing pitch angle.

Figure $2 \mathrm{~b}$. Shown is the NPA measurement of the anisotropic NB ion distribution versus energy and NPA tangency radius after approximately one slowing down time. Compared to Figure $2 \mathrm{a}$, the beam fractional energy components are not as evident due beam slowing down. Evidence of the spectrum filling in below the critical energy, $E_{\text {crit }} \sim 15 \mathrm{keV}$ due to pitch angle scattering can be seen.

Figure 3. Following onset of the $\mathrm{H}$-mode at $230 \mathrm{~ms}$, the NPA spectrum (top panel) shows significant ion loss only for energies $E_{b} / 2<E \leq E_{b}$ (encircled region). This ion loss starts at $\mathrm{t}=280 \mathrm{~ms}$ concurrently with onset of $\mathrm{n}=2$ mode activity (lower panels).

Figure 4. The MPTS electron density profiles (top panel) correspond to times flagged by color-coded solid circles on the line integral density curve (lower panel). In the electron density profile plot, the shaded region denotes the approximate radial location of the source of the NPA signal. 
Figure 5. The TRANSP code is capable of simulating NPA flux measurements. The measured and simulated energetic ion spectra are compared for times $t=180 \mathrm{~ms}$ preceding the $\mathrm{H}$-mode and $\mathrm{t}=400 \mathrm{~ms}$ during the $\mathrm{H}$-mode (top panel). The spectra are normalized once at $30 \mathrm{keV}$ for the pre $\mathrm{H}$-mode case. Note that for clarity an artificial offset has been applied to the "during $\mathrm{H}$-mode" spectra. As shown in the bottom panel, TRANSP significantly overestimates the neutron yield following MHD onset during the $\mathrm{H}$-mode phase.

Figure 6. The magnitude of the energetic ion loss (decrease of $S_{N P A}$ in the region $E_{b} / 2<E \leq$ $E_{b}$ ) was observed to diminish with increasing tangency radius, $R_{t a n}$, of the NPA sightline and with increasing NB injection energy, $E_{b}$.

Figure 7. The NPA signal is localized to a certain extent by beam neutrals. In this case, approximately $2 / 3$ of the integrated flux originates from the NPA/NB intersection region where ions are deposited on passing orbits. The remainder originates in the outboard region of the plasma where ions are more likely to be deposited on trapped orbits. Localization of the NPA signal weakens with decreasing NPA tangency radius due to attenuation of the beam neutrals.

Figure 8. Variation of pitch sampled by the NPA as a function of viewing tangency radius is shown. For a large part of the NPA scan range, $R_{\tan }, \sim 30-120 \mathrm{~cm}$, the diagnostic signal is weighted towards passing ions.

Figure 9. TRANSP analysis suggests that $\mathrm{H}$-mode operation enhances MHD-induced energetic ion loss. The pressure profile generated by the $\mathrm{H}$-mode evolves the q-profile to permit low-n MHD activity (right column) while shifting neutral beam deposition into the MHD active region (left column). This synergism between MHD activity and outward shift of the neutral beam deposition leads to the enhanced energetic ion loss observed in $\mathrm{H}$-mode discharges. 
Figure 10. A discharge is shown that definitively demonstrates the synergism between MHD activity and outward shift of the neutral beam deposition that leads to enhanced energetic ion loss in $\mathrm{H}$-mode discharges. It can be seen that the $\mathrm{n}=1,2,3$ activity setting in at $300 \mathrm{~ms}$ appears to have little or no effect on the neutron and NPA signals. However, at the $\mathrm{H}$-mode transition a marked decrease of the NPA signal occurs followed by a drop in the neutron rate. Thus is can be concluded that MHD activity alone does not induce significant ion loss but that MHD activity in conjunction with $\mathrm{H}$-mode does.

Figure 11. Results of NPA signal flux modeling for two injection energies $E_{b}=80,40 \mathrm{keV}$ are shown. The modeled flux is displayed as a function of mode amplitude $\square_{0}(\mathrm{a})$ and the scaling factor of the q-profile (b). ORBIT code modeling shows that resonant loss occurs for $E>E_{b} / 2$ but not for lower energies, in agreement with NPA measurements.

Figure 12. ORBIT modeling shows that resonant loss of order $10 \%-15 \%$ occurs for $E>$ $E_{b} / 2$ but not for lower energies, in agreement with NPA observations.

Figure 13. The measured neutron rate and simulated neutron rates with fast ion diffusion (108730M07) and without fast ion diffusion (108730M08) are shown. The fast ion diffusion in TRANSP is increased so as to match the calculated and measured neutron rates.

Figure 14. Comparison of TRANSP ion spectra with and without fast ion diffusion against NPA measurements for SN108730 at $t=400 \mathrm{~ms}$.

Figure 15. TRANSP calculations of neutral beam power to electrons and ions (upper panel) for SN108730 without energetic ion diffusion (blue curves) and with energetic ion diffusion (red curves) and NB fast ion power loss (lower panel) are shown.

Figure 16. TRANSP calculation of ion power balance at $t=400 \mathrm{~ms}$ with and without fast ion diffusion for $\mathrm{SN} 108730$ is shown. $I_{\text {heat }}$ is the total ion heating, $Q_{i e}$ is the power 
transferred from ions to electrons (which is negative because $T_{i}>T_{e}$ ) and $P_{\text {condi }}$ is the ion conduction power loss.

Figure 17. TRANSP calculation of electron power balance at $t=400 \mathrm{~ms}$ with and without fast ion diffusion for $\mathrm{SN108730}$ is shown. $E_{\text {heat }}$ is the total electron heating, $Q_{i e}$ is the power transferred from ions to electrons (which is positive because $T_{i}>T_{e}$ ) and $P_{\text {conde }}$ is the electron conduction power loss.

Figure 18. TRANSP calculation of power balance diffusivities at $t=400 \mathrm{~ms}$ with and without fast ion diffusion for SN108730 is shown. In the core region, $\square_{\square} \ll \square_{e} \square$ [ist $^{\text {ast }}<$ $\square_{i} \square i^{N C}$ with and without fast ion diffusion. In the mid-radius region, $\square_{\square}<\square_{i}<\square_{i}^{N C} \sim$ $\mathrm{C}_{\mathrm{e}} \backslash \square^{\mathrm{Fast}}$ both with and without the fast ion diffusion model, though it can be seen that fast ion diffusion modestly reduces $\square_{\mathrm{e}}$ but markedly reduces $\square_{\mathrm{i}}$.

Figure 19. TRANSP calculation of thermal energy confinement time (a) and toroidal beta (b) at $\mathrm{t}=400 \mathrm{~ms}$ with and without fast ion diffusion for SN108730 is shown. For this case, accounting for energetic ion loss increased the thermal energy confinement, $\mathrm{L}$, by $\sim 15 \%$ and reduced the toroidal beta, $\square_{\mathrm{T}}$, by $\sim 7 \%$.

Figure 20. The NPA energetic ion spectrum and its approximation at high energy by the slowing down distribution without loss (solid line) and with finite loss time (dashed line) is shown. From analysis of the energy spectrum, the energetic ion loss time can be estimated to be on the order of $4 \mathrm{~ms}$. 


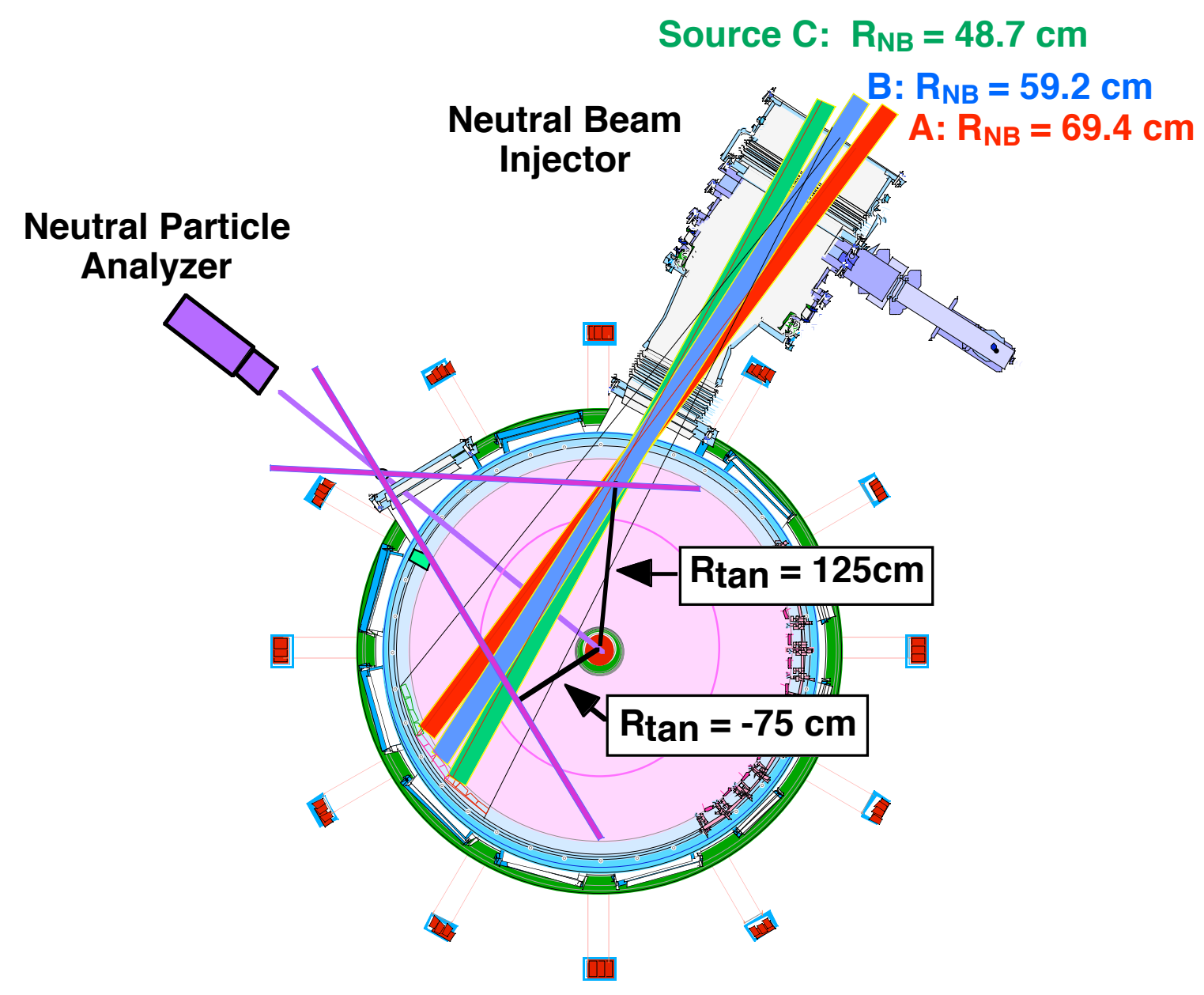

Figure 1. The Neutral Particle Analyzer (NPA) on NSTX views across the three neutral beam injection sources and can be scanned over a wide range of sightline tangency radii, $R_{\text {tan, }}$ on a shot-to-shot basis. $R_{\text {tan }}$, is the perpendicular distance between the machine center and the NPA sightline. 


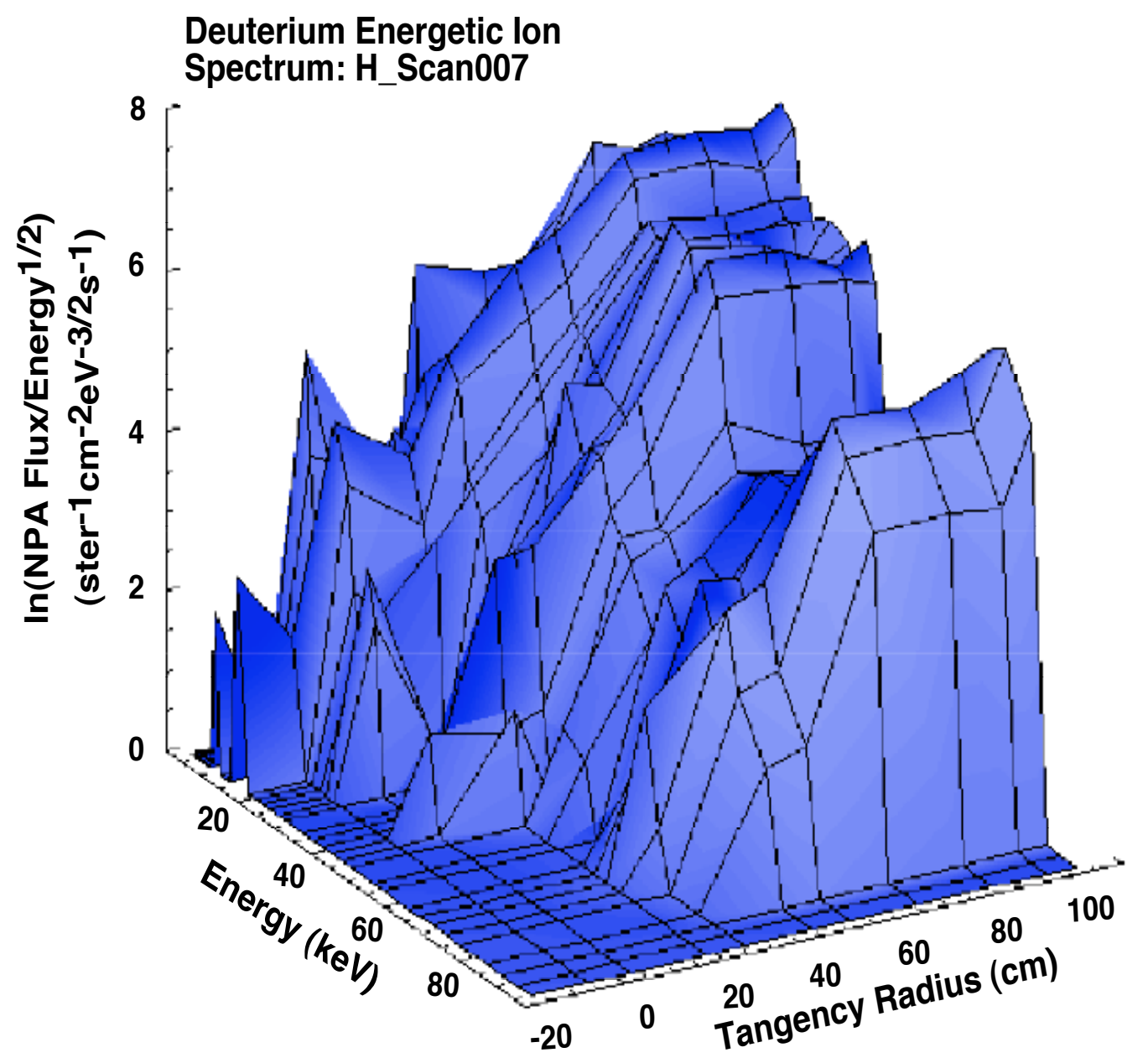

Figure 2a. Shown is the NPA measurement of the NB ion distribution versus energy and NPA tangency radius or near the start of neutral beam injection. The spectrum peaks around the beam injection tangency radius, $R_{N B} \sim 0.6$ $m$, and the full, half and third beam energy components are evident. The spectrum is highly anisotropic with the ion population depleting with decreasing NPA tangency radius, which corresponds to decreasing pitch angle. 


\section{0 msec later}

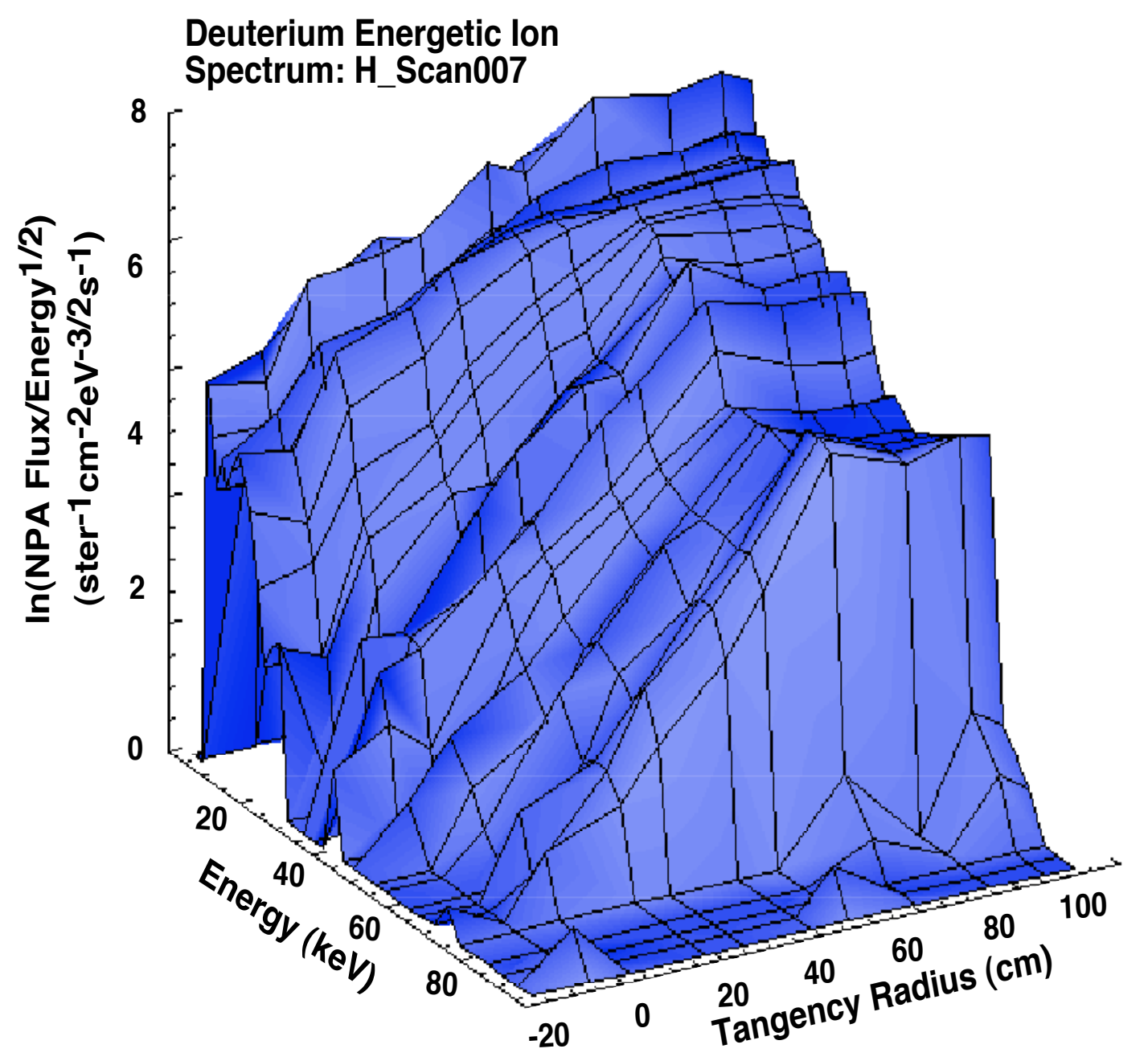

Figure 2b. Shown is the NPA measurement of the anisotropic NB ion distribution versus energy NPA tangency radius after approximately one slowing down time. Compared to Figure 2a, the beam fractional energy components are not as evident due beam slowing down. Evidence of the spectrum filling in below the critical energy, $E_{\text {crit }} \sim 15 \mathrm{keV}$ due to pitch angle scattering can be seen. 

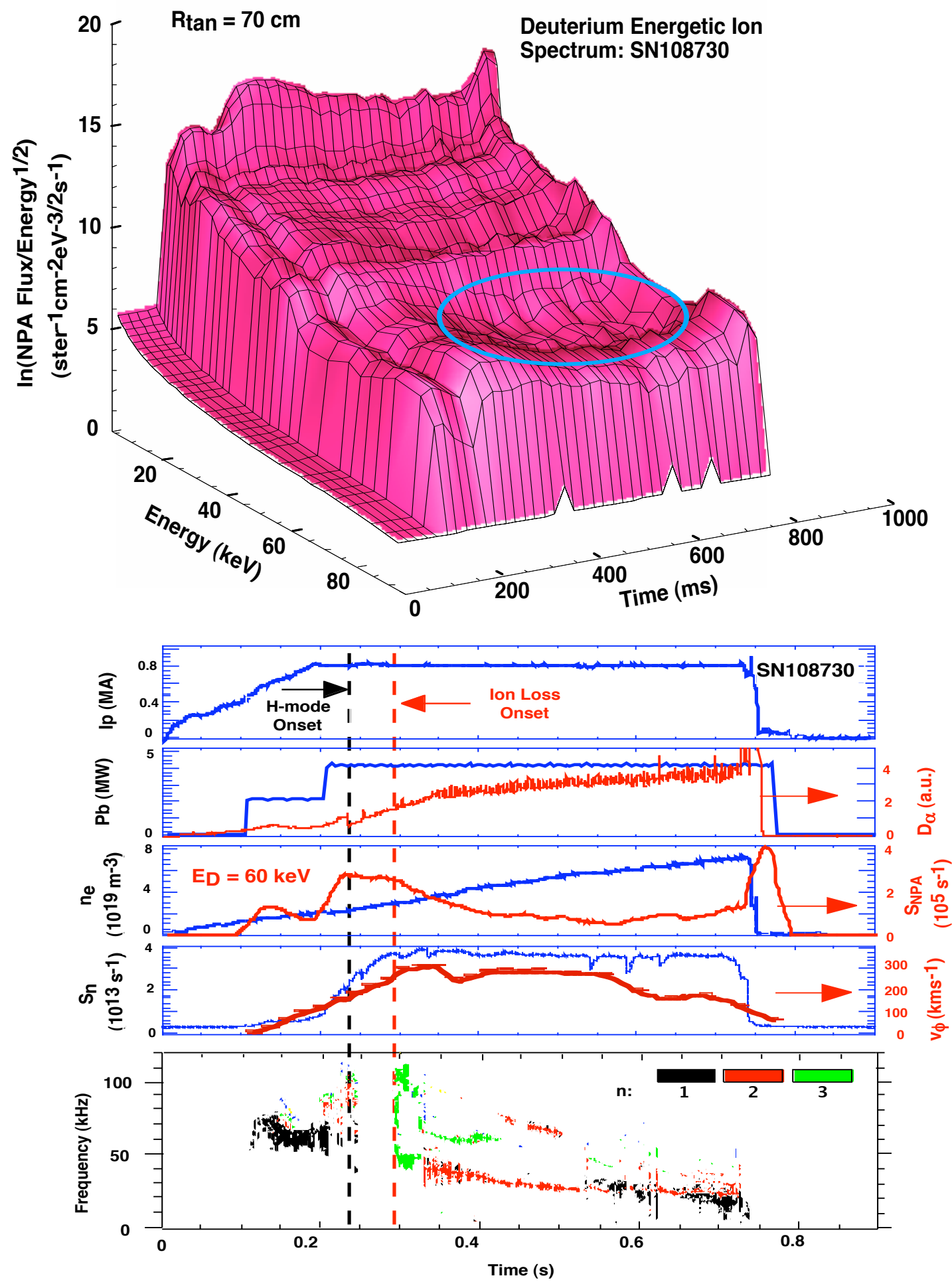

Figure 3. Following onset of the $\mathrm{H}$-mode at $230 \mathrm{~ms}$, the NPA spectrum (top panel) shows significant ion loss only for energies $E_{b} / 2<E \leq E_{b}$ (encircled region). This loss starts at $t=280 \mathrm{~ms}$ concurrently with onset of $n=2$ mode activity (lower panels). 

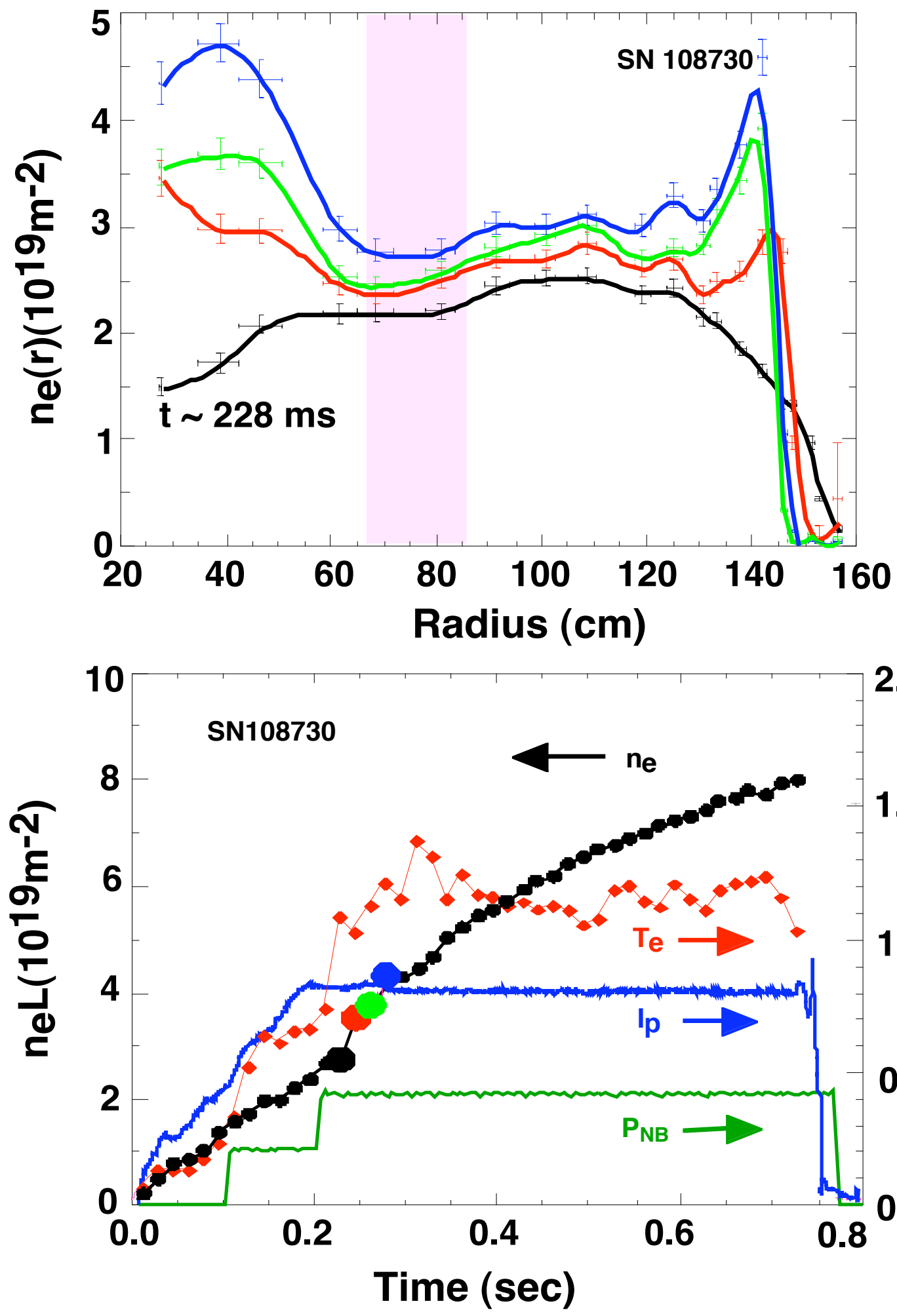

2.0

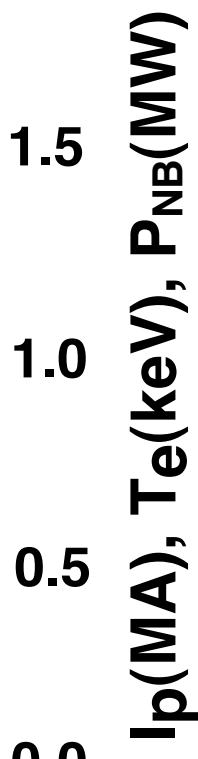

0.0

Figure 4. The MPTS electron density profiles (top panel) correspond to times flagged by color-coded solid circles on the line integral density curve (lower panel). In the electron density profile plot, the shaded region denotes the approximate radial location of the source of the NPA signal. 

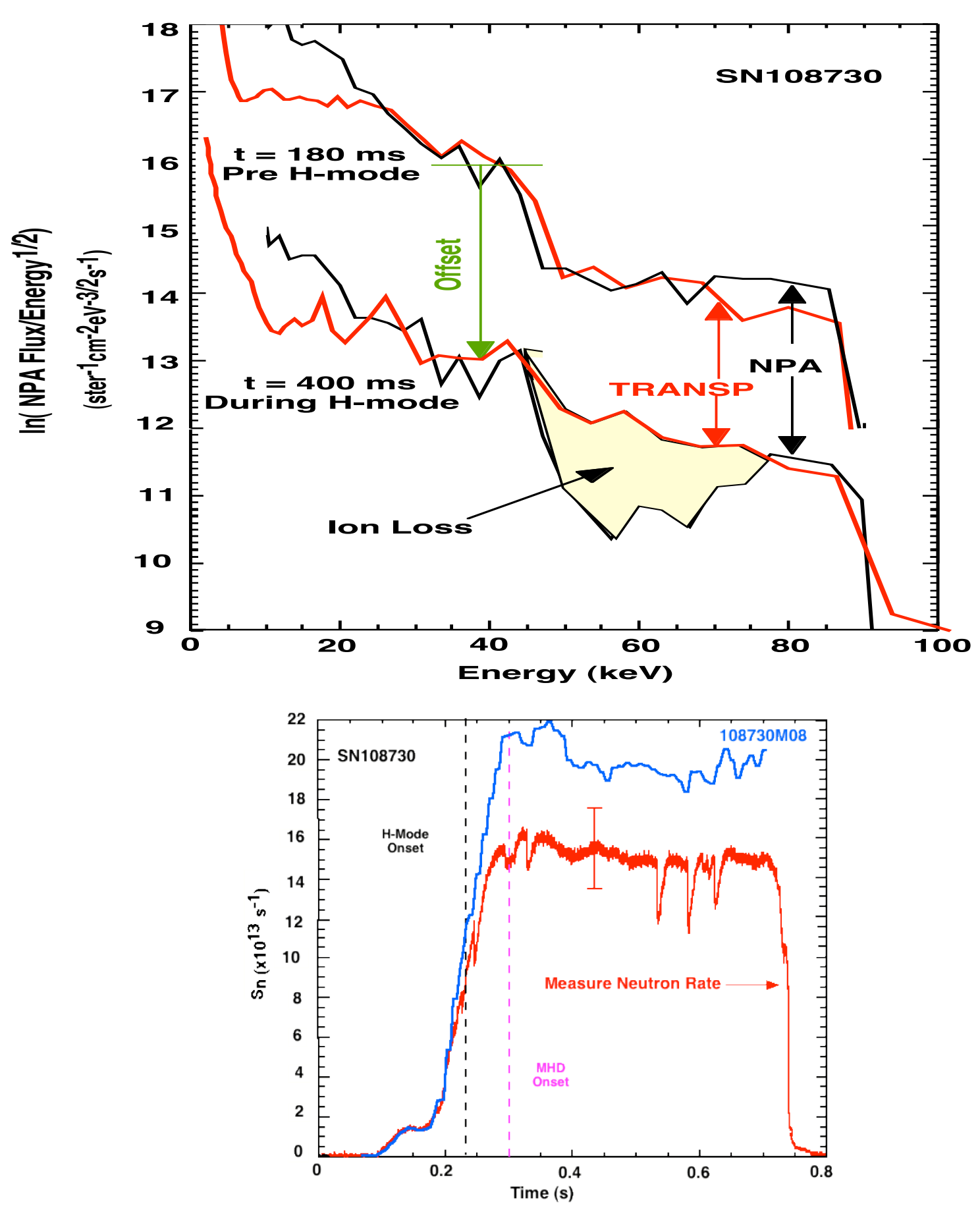

Figure 5. The TRANSP code is capable of simulating the NPA flux measurements. The measured and simulated energetic ion spectra are compared for times $t=180 \mathrm{~ms}$ preceding the $\mathrm{H}$-mode and $t=400 \mathrm{~ms}$ during the $\mathrm{H}$-mode (top panel). The spectra are normalized once at $30 \mathrm{keV}$ for the pre $\mathrm{H}$-mode case. Note that for clarity an artificial offset has been applied to the "during H-mode" spectra. As shown in the bottom panel, TRANSP significantly overestimates the neutron yield following MHD onset during the H-mode phase. 

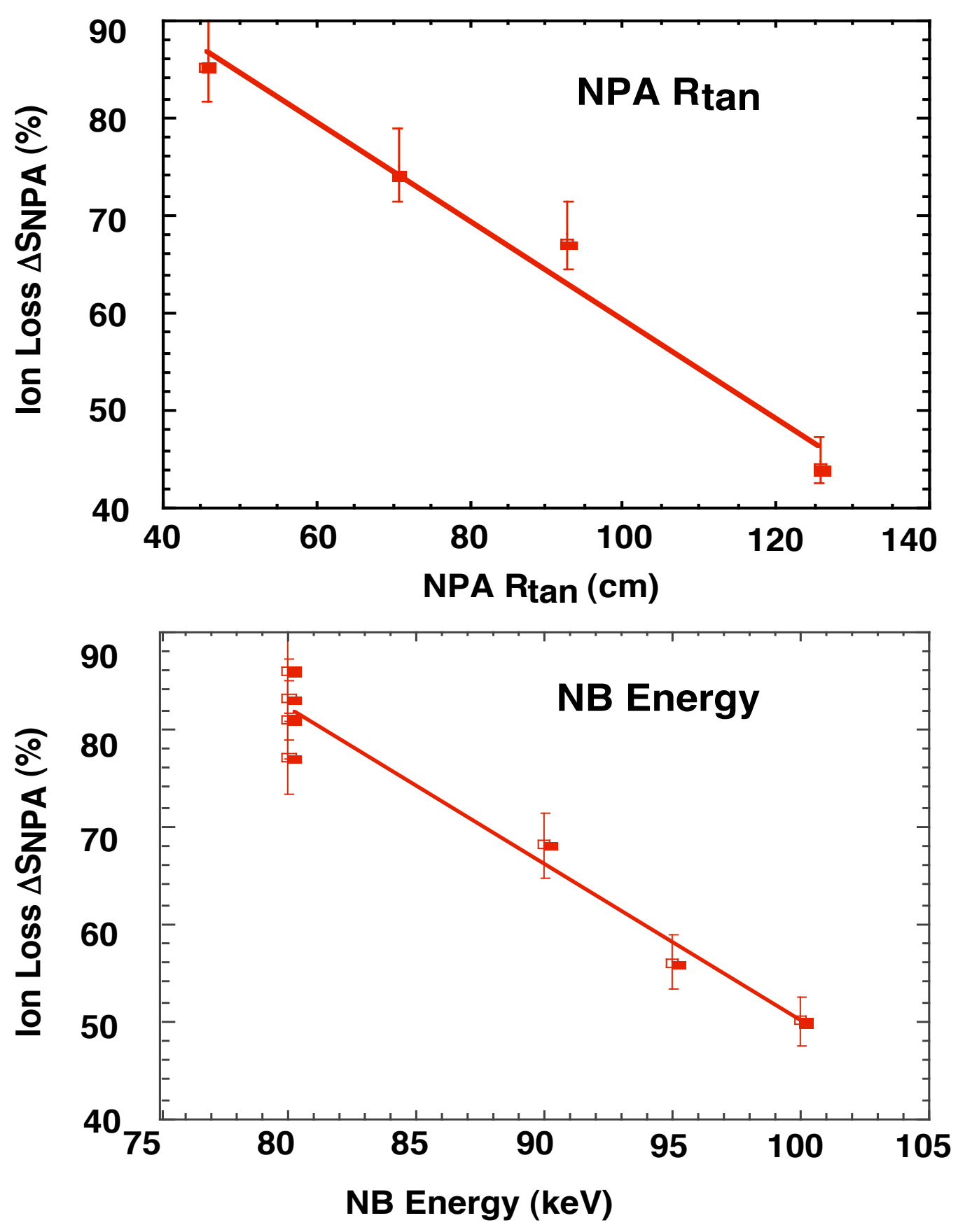

Figure 6. The magnitude of the energetic ion loss (decrease of $S_{N P A}$ in the region $E_{b} / 2<E \leq E_{b}$ ) was observed to diminish with increasing tangency radius, $R_{\text {tan }}$, of the NPA sightline and with increasing NB injection energy, $E_{b}$. 


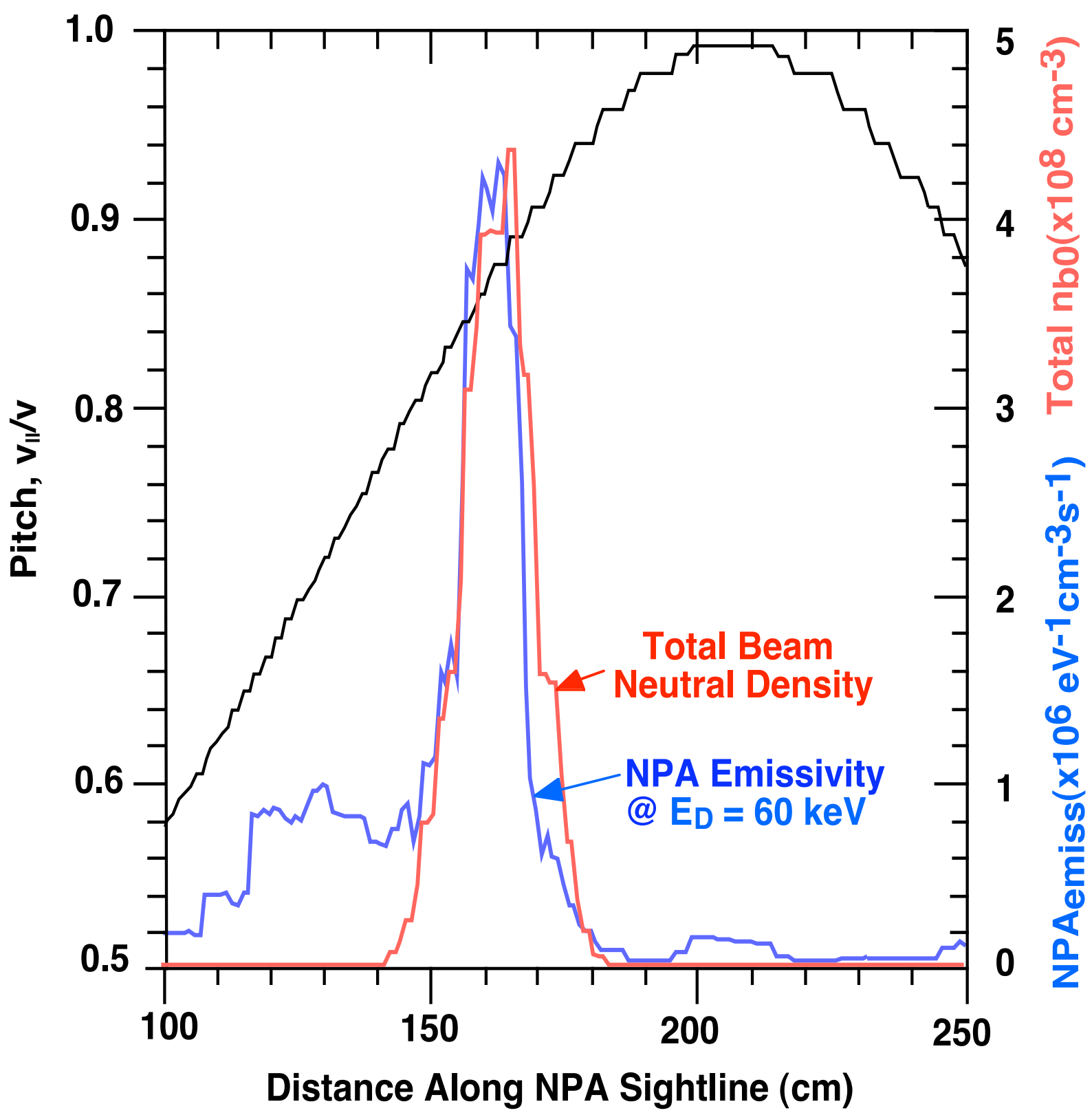

Figure 7. The NPA signal is localized to a certain extent by beam neutrals. In this case, approximately $2 / 3$ of the integrated flux originates from the NPA/NB intersection region where ions are deposited on passing orbits. The remainder originates in the outboard regions of the plasma where ions are more likely to be deposited on trapped orbits. Localization of the NPA signal weakens with decreasing NPA tangency radius due to attenuation of the beam neutrals. 


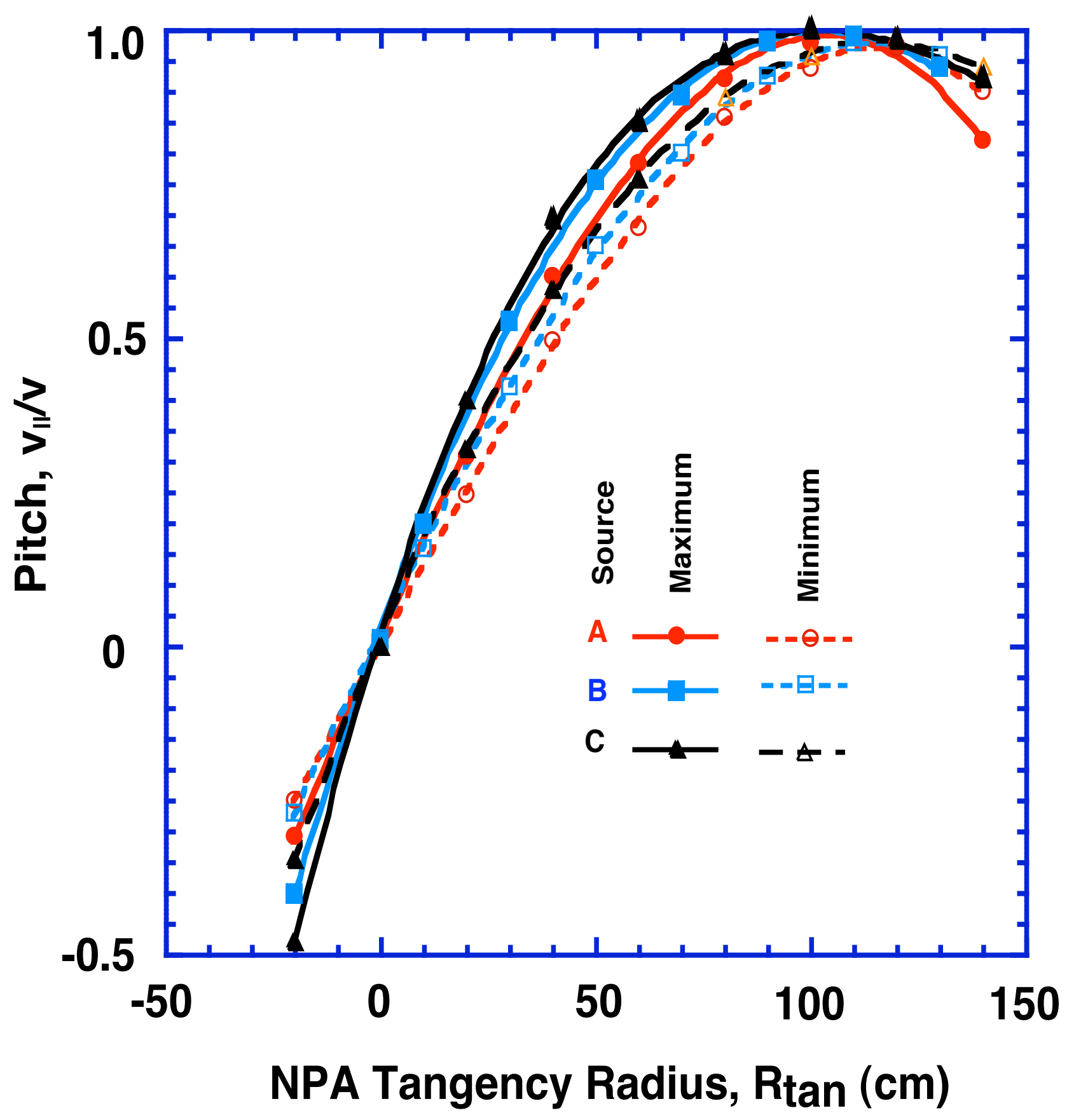

Figure 8. Variation of pitch sampled by the NPA as a function of viewing tangency radius is shown. For a large part of the NPA scan range, $R_{\text {tan }}, \sim$ $30-120 \mathrm{~cm}$, the diagnostic signal is weighted towards passing ions. 

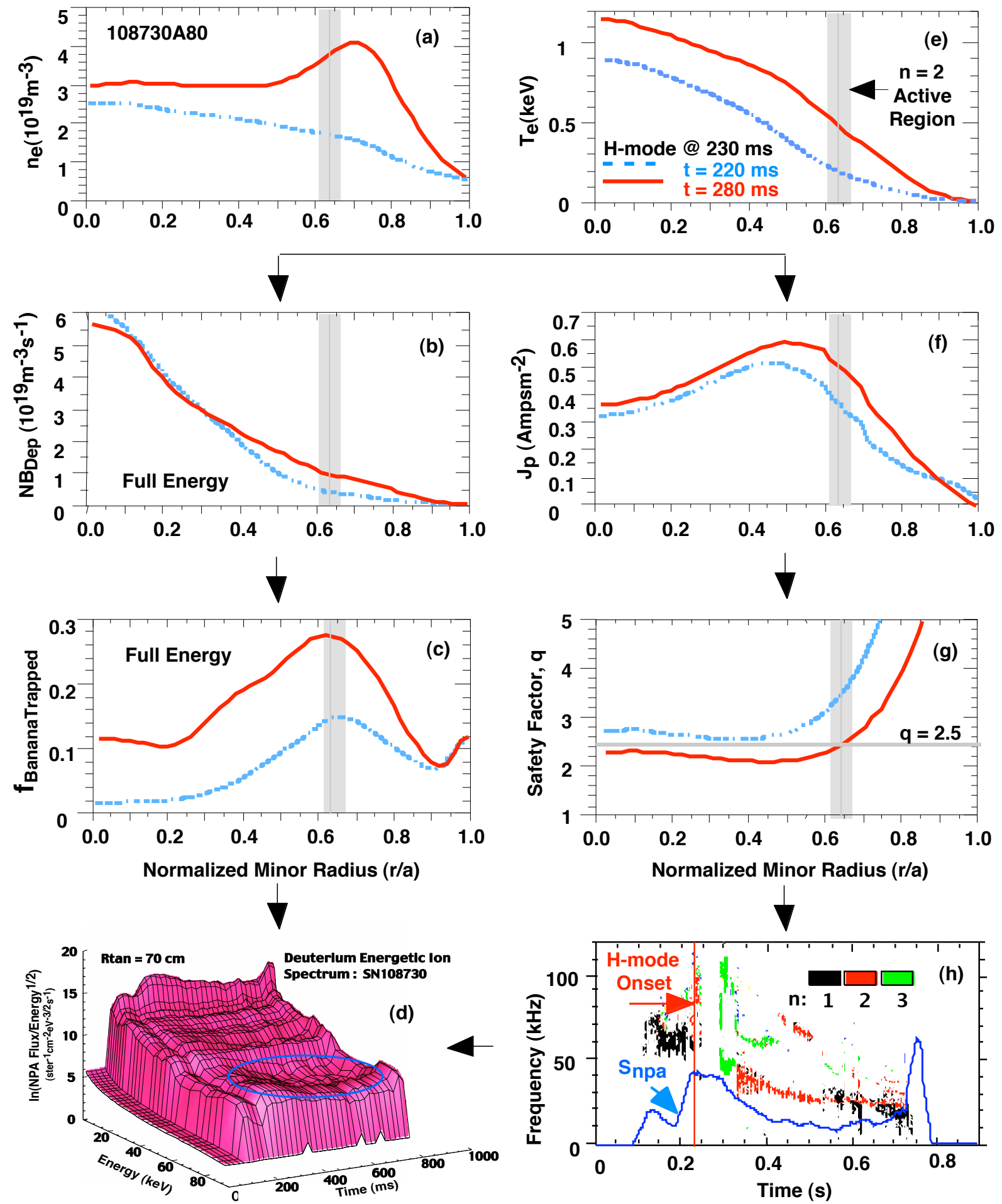

Figure 9. TRANSP analysis suggests that $\mathrm{H}$-mode operation enhances MHD-induced energetic ion loss. The pressure profile generated by the $\mathrm{H}$-mode evolves the q-profile to permit low- $n$ MHD activity (right column) while shifting neutral beam deposition into the MHD active region (left column). This synergism between MHD activity and outward shift of the neutral beam deposition leads to enhanced energetic ion loss observed in $\mathrm{H}$-mode discharges. 


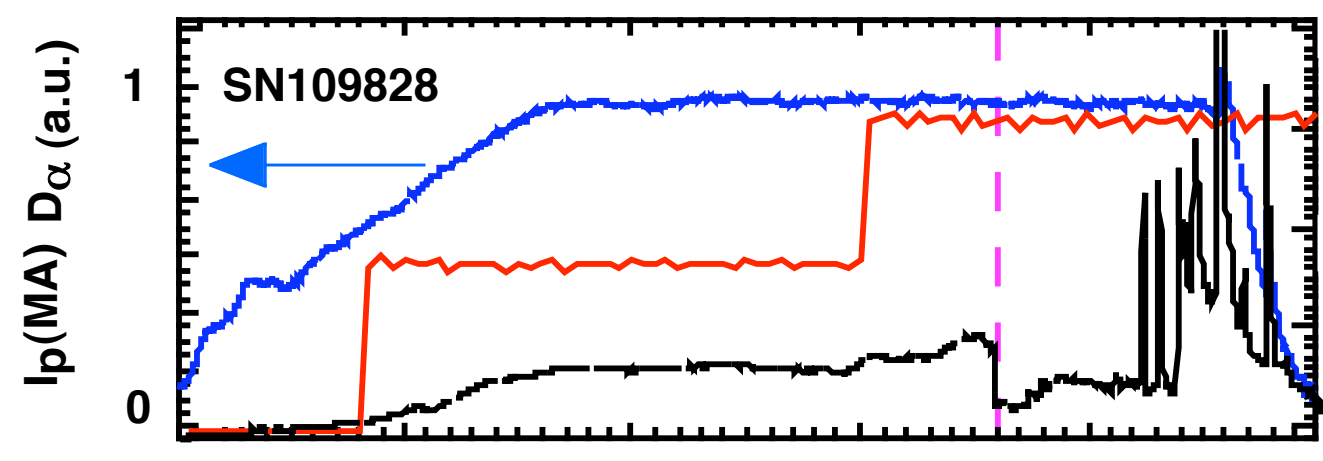

$2 \sum_{\frac{m}{z}}^{\sum}$

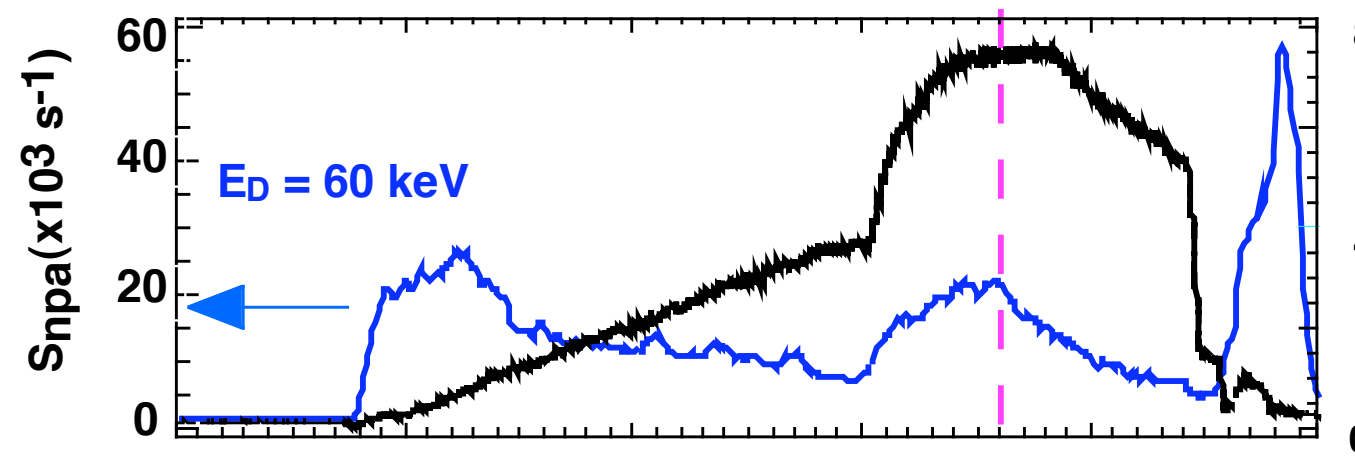

0

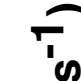

4 $\frac{m}{0}$
$\frac{0}{x}$
क

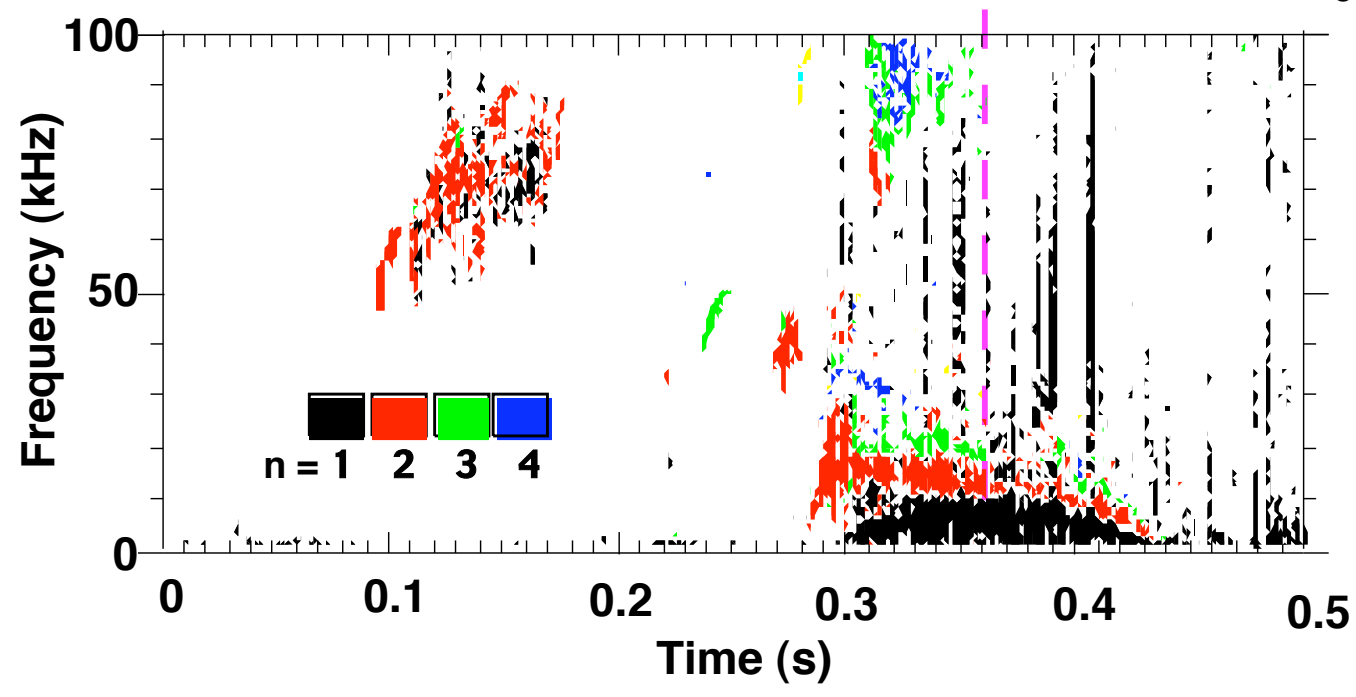

Figure 10. A discharge is shown that definitively demonstrates the synergism between MHD activity and outward shift of the neutral beam deposition that leads to enhancea energetic ion loss in $\mathrm{H}$-mode discharges. It can be seen that the $n=1,2,3$ activity that sets in at 300 ms appears to have little or no effect on the neutron and NPA signals. However, at the H-mode transition a marked decrease of the NPA signal occurs followed by a drop in the neutron rate. Thus is can be concluded that MHD activity alone does not induce significant ion loss but that MHD activity in conjunction with $\mathrm{H}$ mode does. 

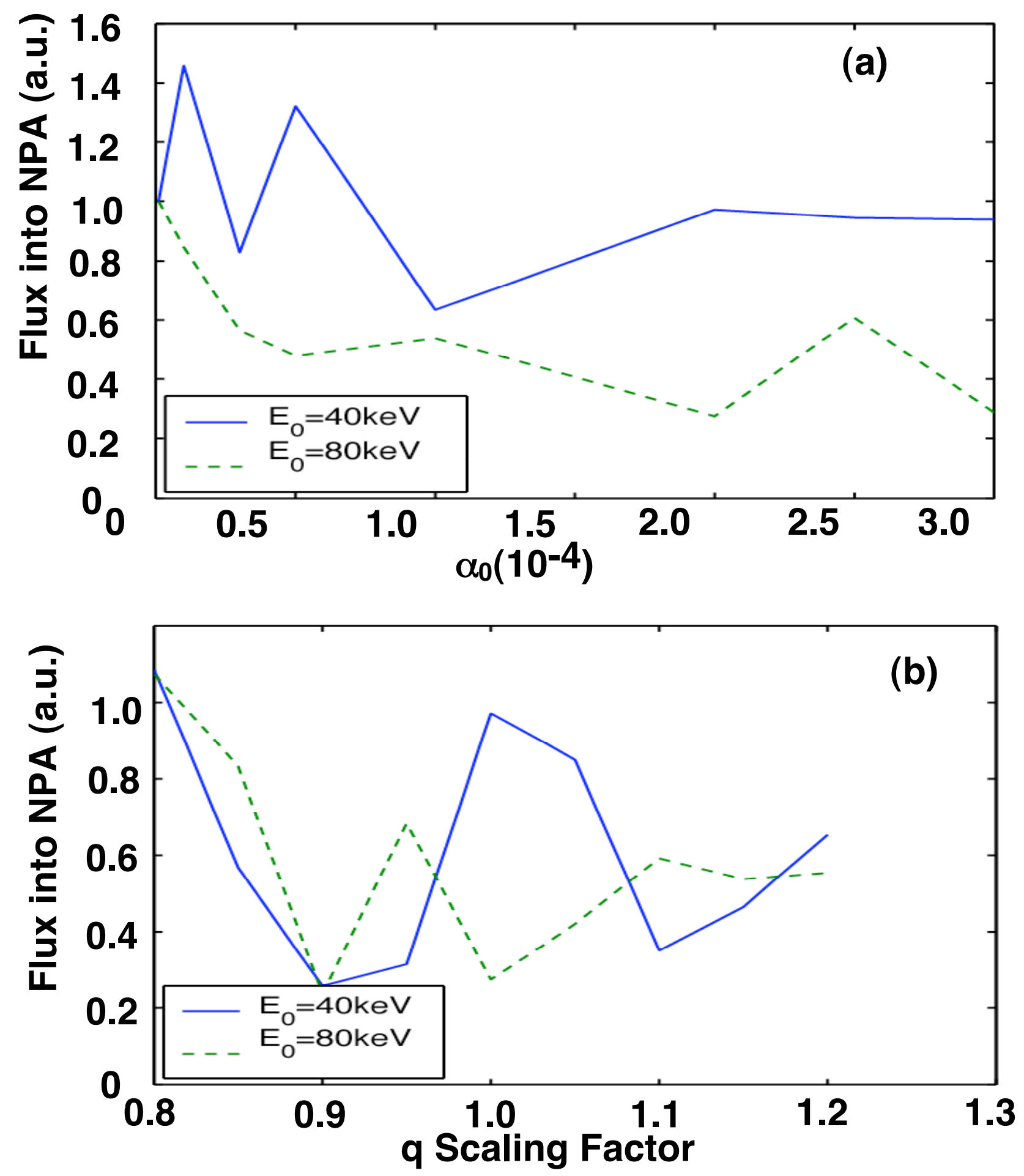

Figure 11. Results of NPA signal flux modeling for two injection energies $E_{b}=80,40 \mathrm{keV}$ are shown. The modeled flux is displayed as a function of mode amplitude $\square_{0}$ (a) and the scaling factor of the q-profile (b). ORBIT code modeling shows that resonant loss occurs for $E>E_{b} / 2$ but not for lower energies, in agreement with NPA measurements 


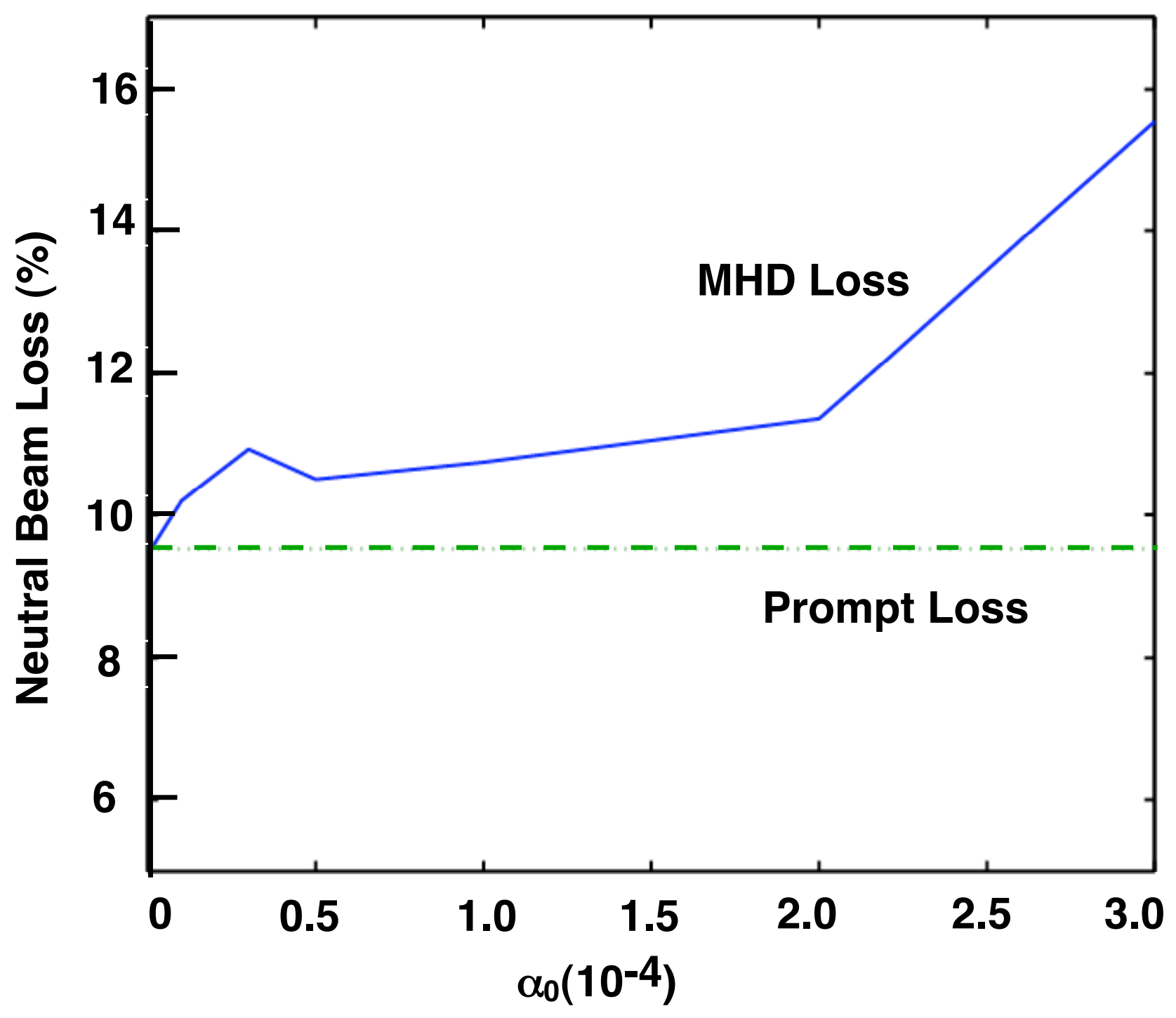

Figure 12. ORBIT modeling shows that resonant loss of order $10 \%$ $15 \%$ occurs for $E>E_{b} / 2$ but not for lower energies, in agreement with NPA observations. 


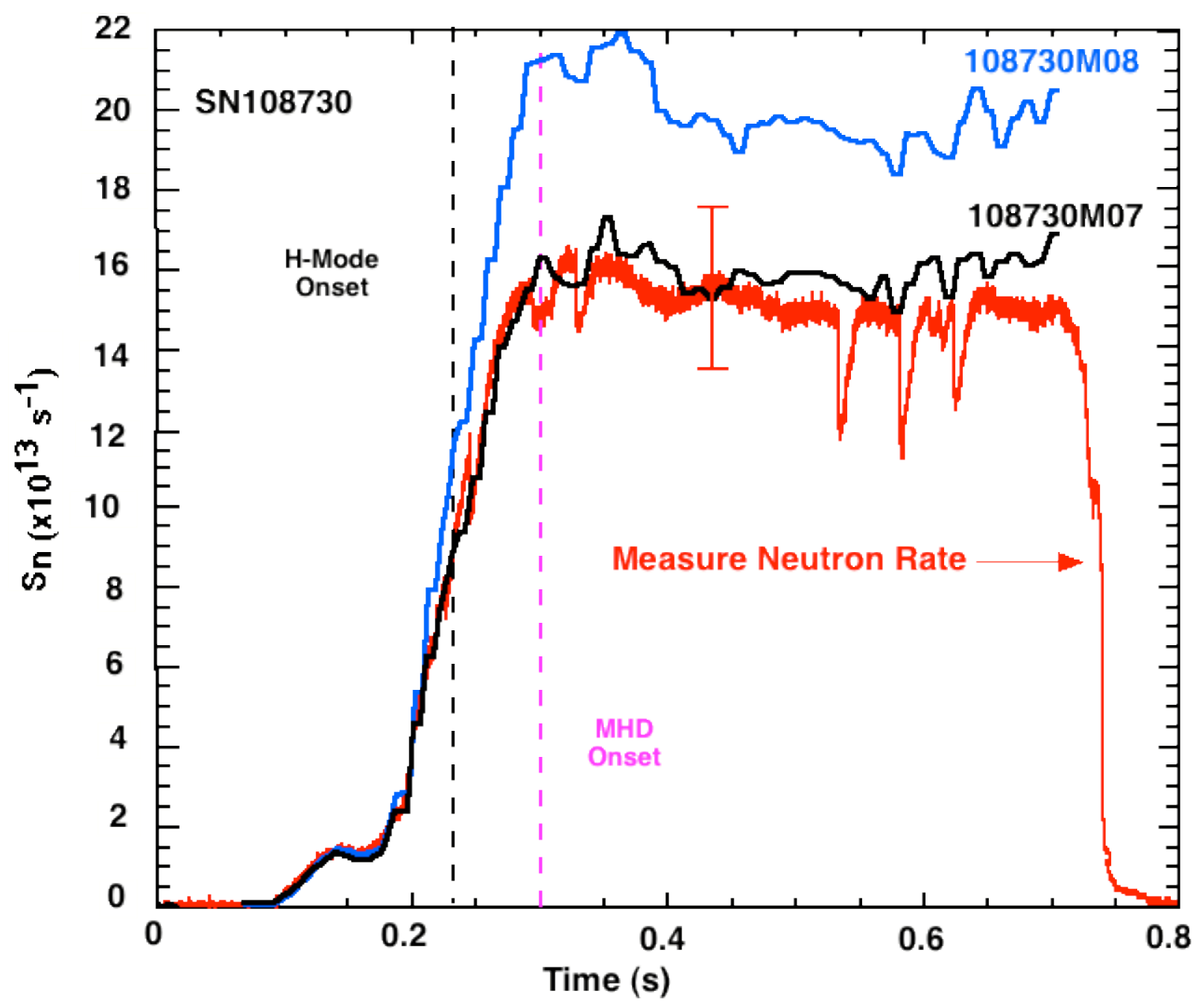

Figure 13. The measured neutron rate and simulated neutron rates with fas ion diffusion (108730M07) and without fast ion diffusion (108730M08) art shown. The fast ion diffusion in TRANSP is increased so as to match the calculated and measured neutron rates. 


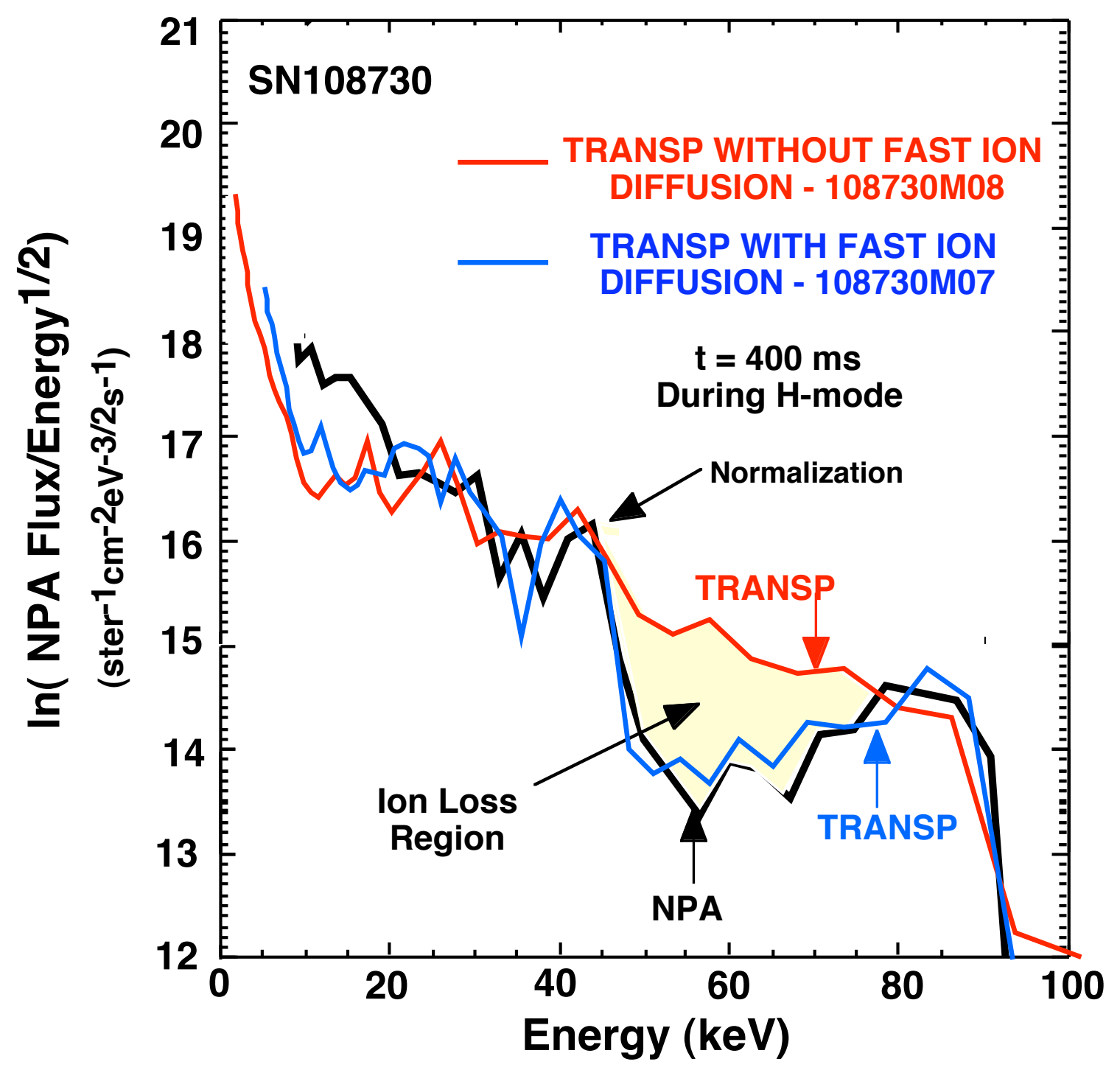

Figure 14. Comparison of TRANSP ion spectra with and without fast ion diffusion against NPA measurements for SN108730 at $t=400 \mathrm{~ms}$. 


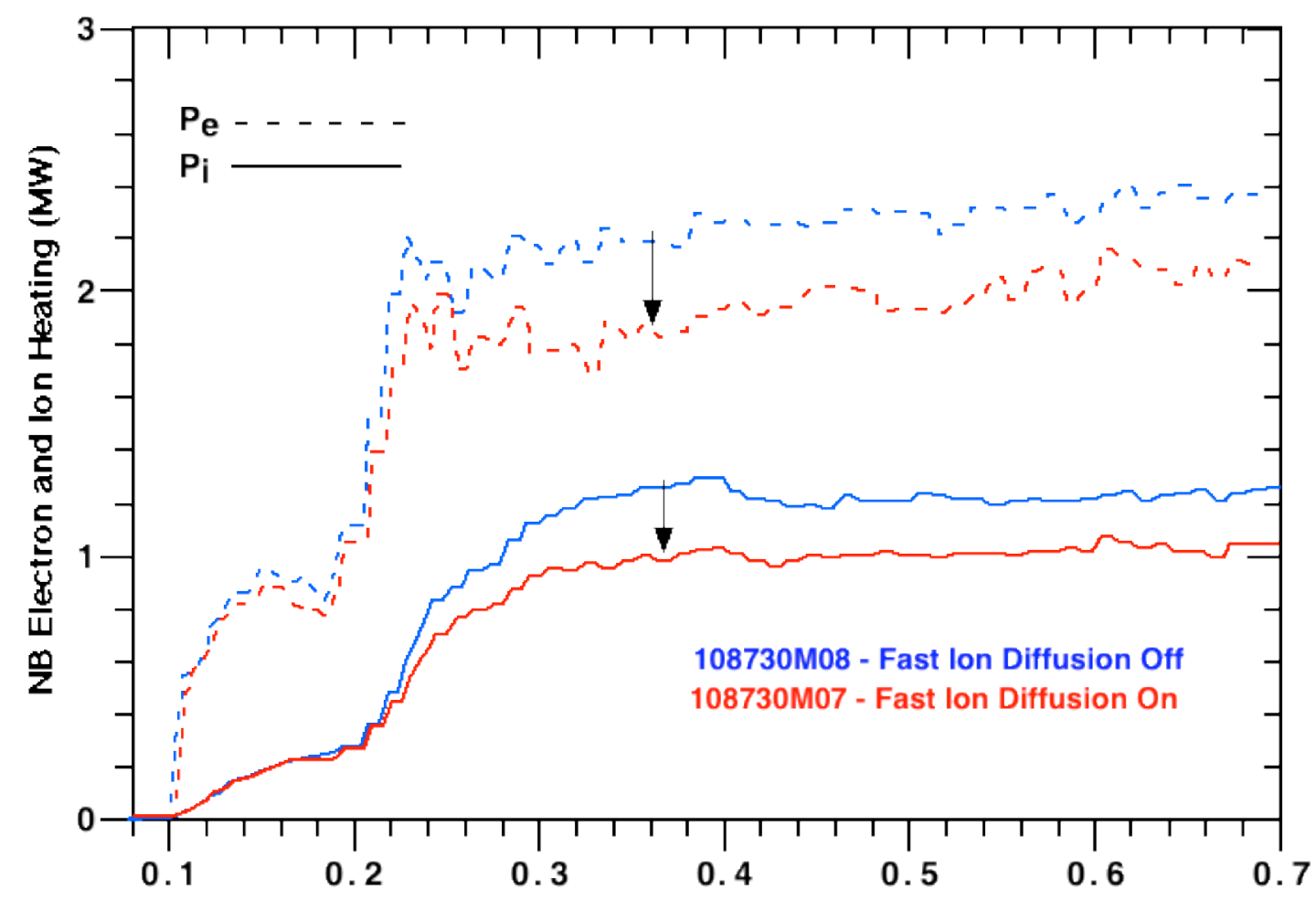

Time (s)

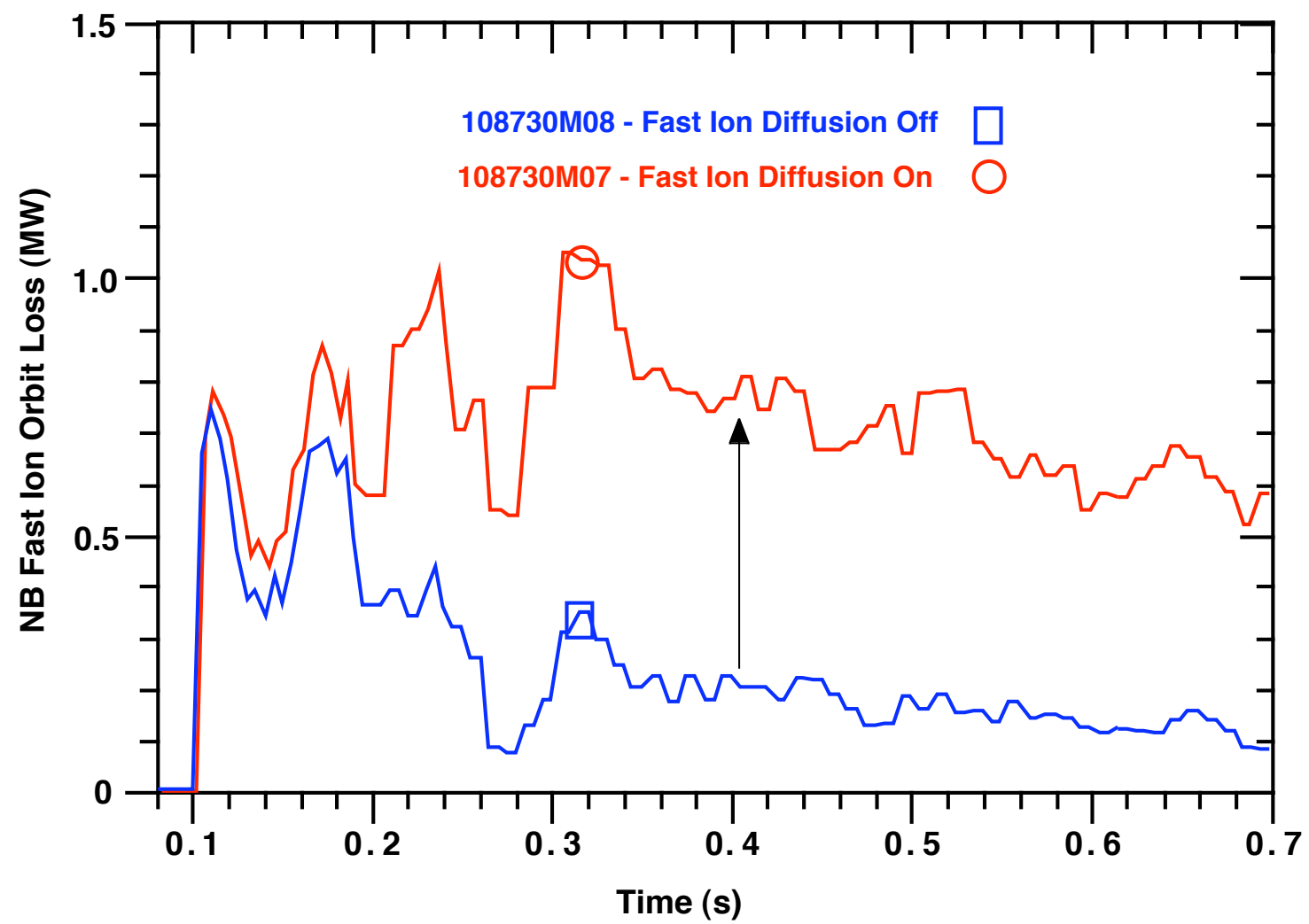

Figure 15. TRANSP calculations of neutral beam power to electrons and ions (upper panel) for SN108730 without fast ion diffusion (blue curves) and with fast ion diffusion (red curves) and NB fast ion power loss (lower panel) are shown. 


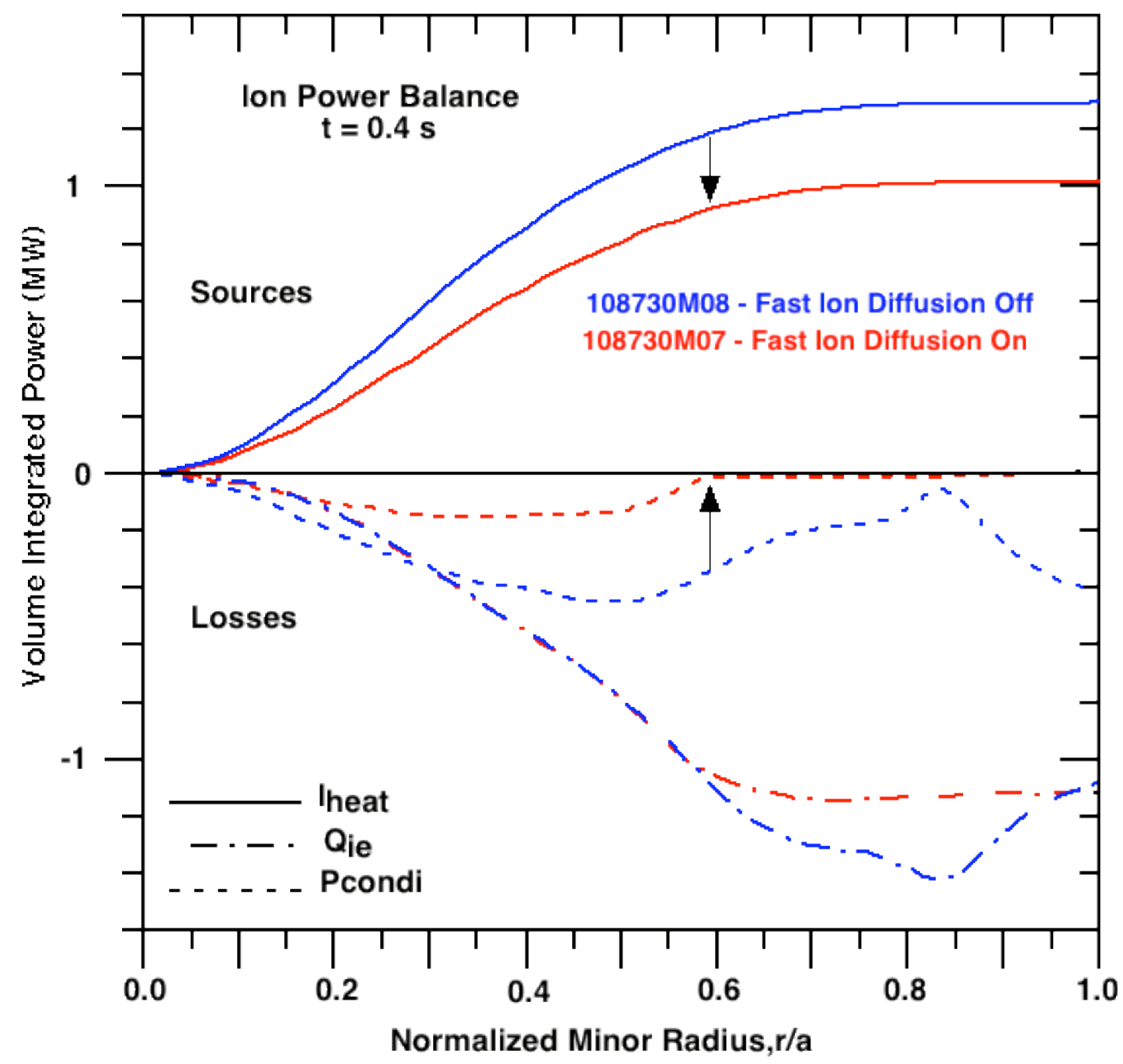

Figure 16. TRANSP calculation of ion power balance at $t=400 \mathrm{~ms}$ with and without fast ion diffusion for SN108730 is shown. $I_{\text {heat }}$ is the total ion heating, $Q_{i e}$ is the power transferred from ions to electrons (which is negative because $\left.T_{i}>T_{e}\right)$ and $P_{\text {condi }}$ is the ion conduction power loss. 


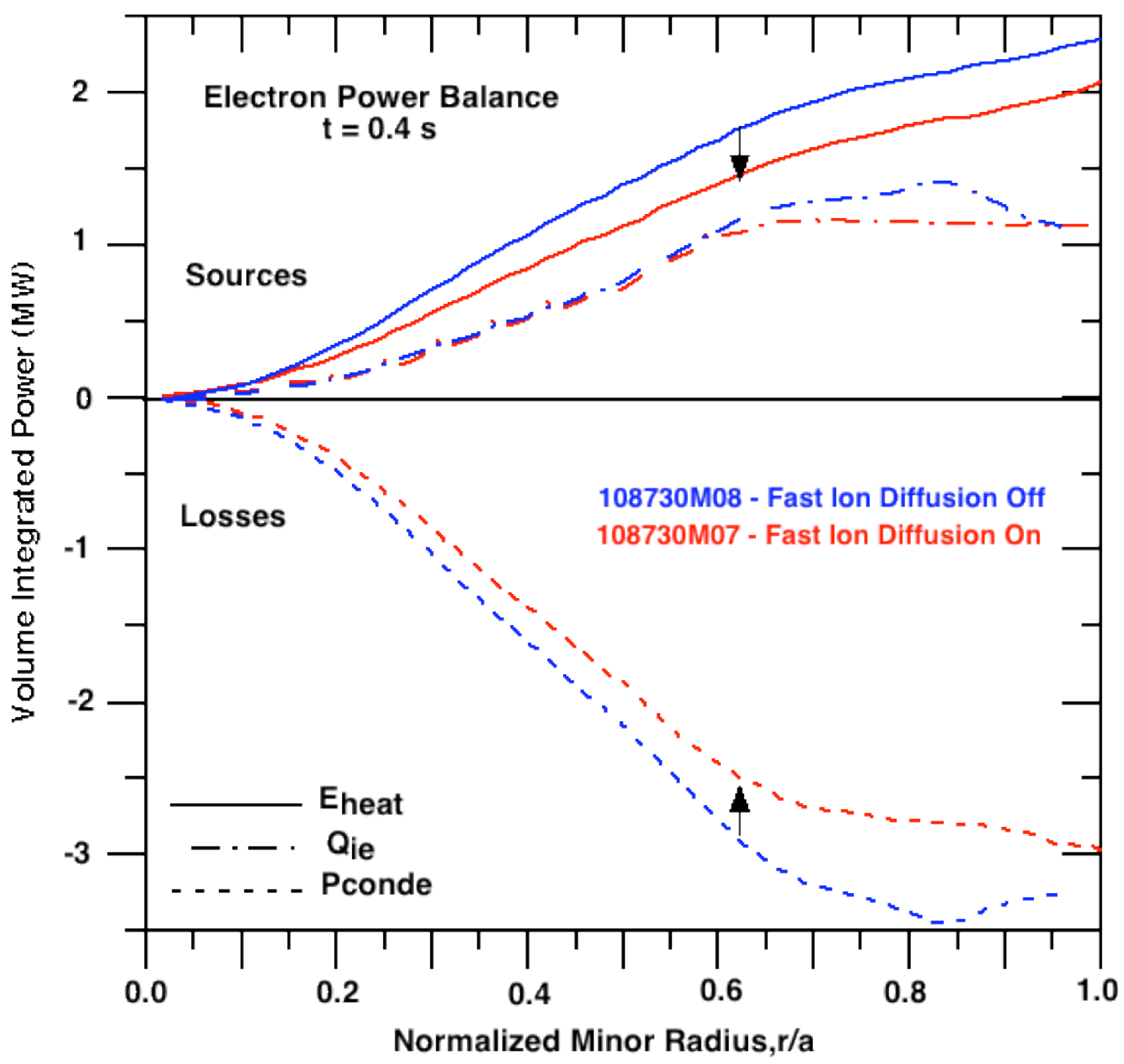

Figure 17. TRANSP calculation of electron power balance at $t=400 \mathrm{~ms}$ with and without fast ion diffusion for SN108730 is shown. $E_{\text {heat }}$ is the total electron heating, $Q_{i e}$ is the power transferred from ions to electrons (which is positive because $T_{i}>T_{e}$ ) and $P_{\text {conde }}$ is the electron conduction power loss. 


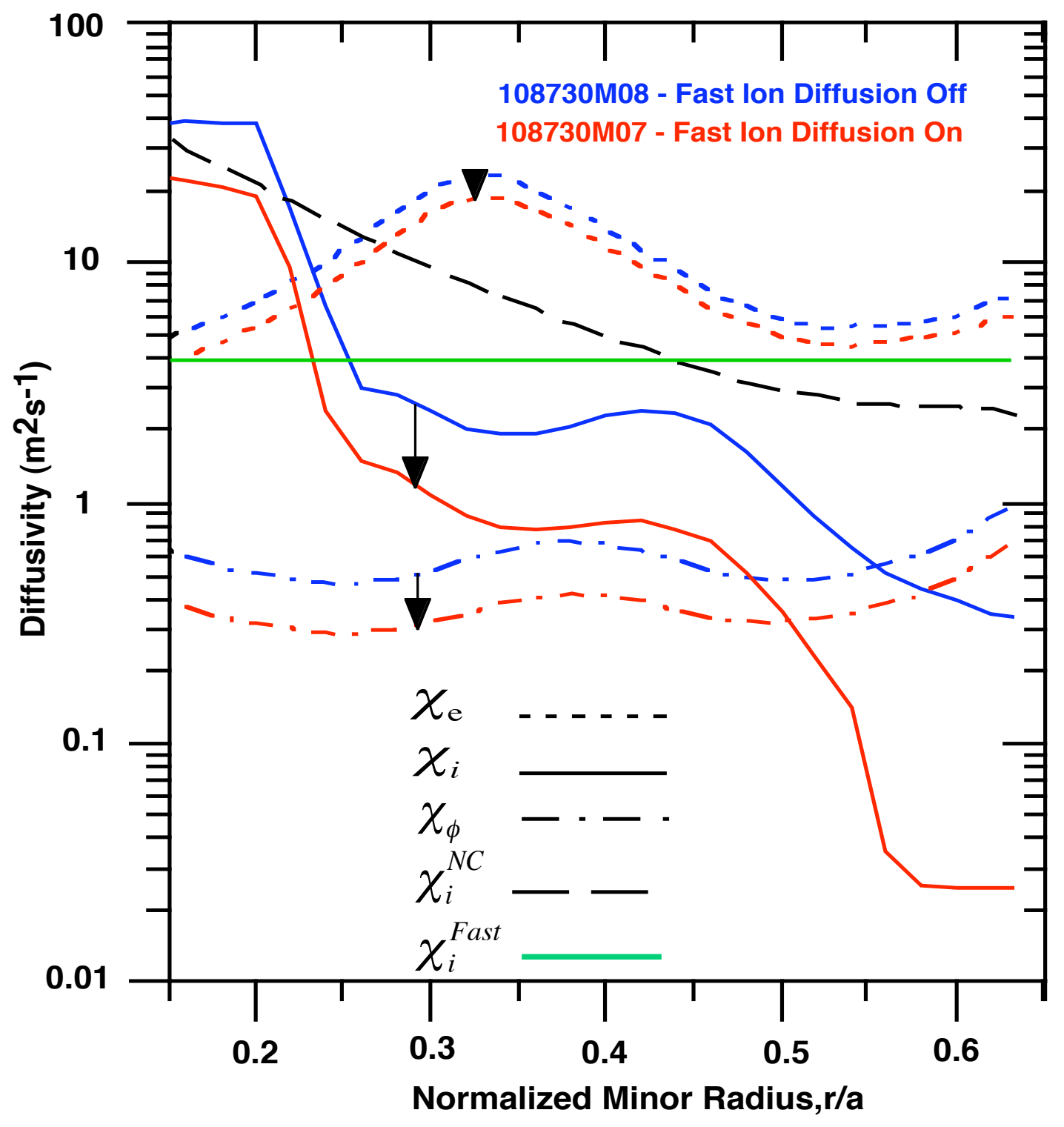

Figure 18. TRANSP calculation of power balance diffusivities at $t=400 \mathrm{~ms}$ with and without fast ion diffusion for SN108730 is shown. In the core region, $\square_{\square}<<\square_{\mathrm{e}} \square \square_{i}^{\mathrm{Fast}}<\square_{\mathrm{i}} \square_{i}^{N C}$ with and without fast ion diffusion. In the mid-radius region, $\square_{\square}<\square_{\mathrm{i}}<\square_{\mathrm{i}}^{\mathrm{NC}} \sim \square_{\mathrm{e}} \square\left[^{\text {ast }}\right.$ both with and without the fast ion diffusion model, though it can be seen that fast ion diffusion modestly reduces $\square_{\mathrm{e}}$ but markedly reduces $\square_{\mathrm{i}}$. 


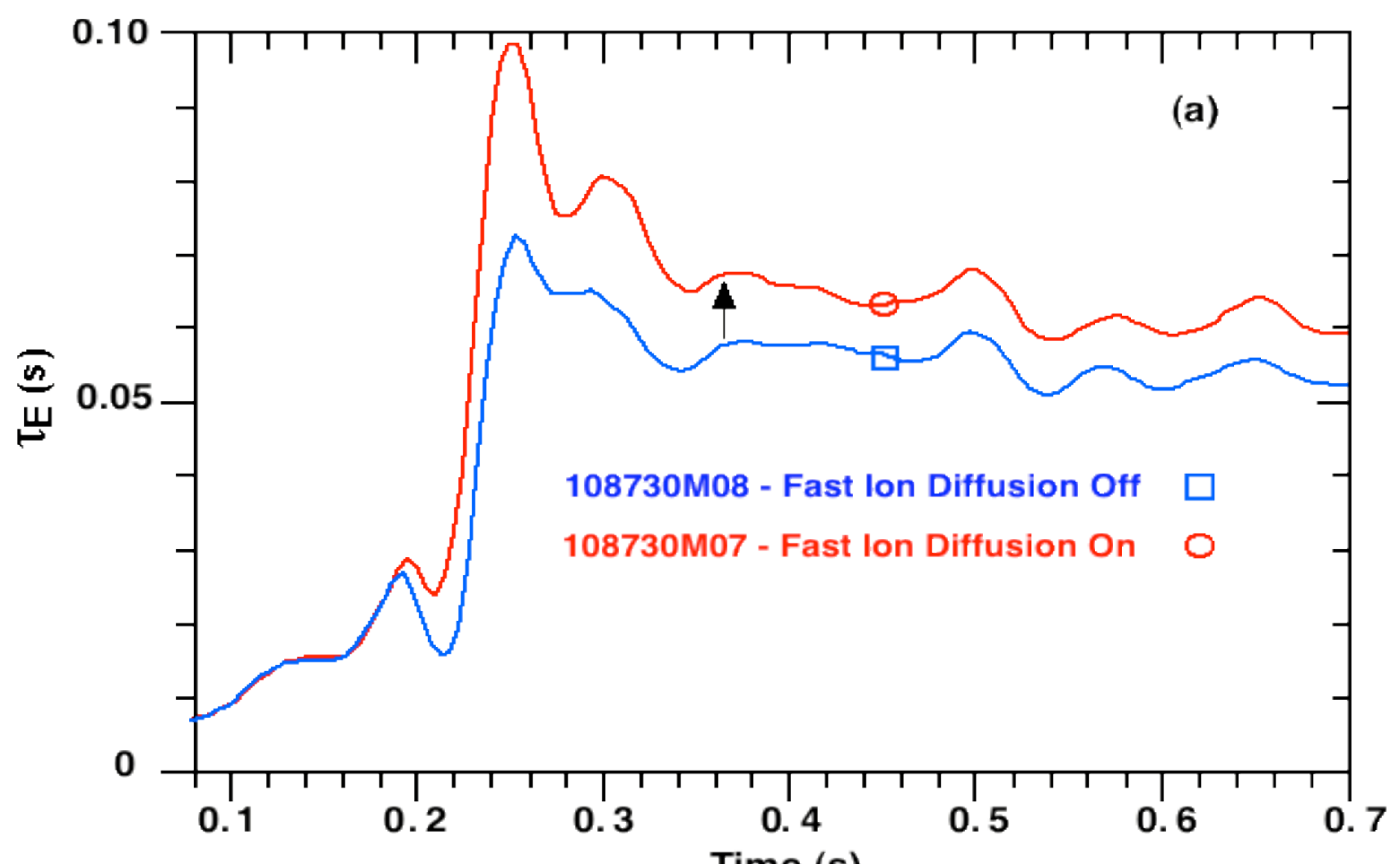

Time (s)

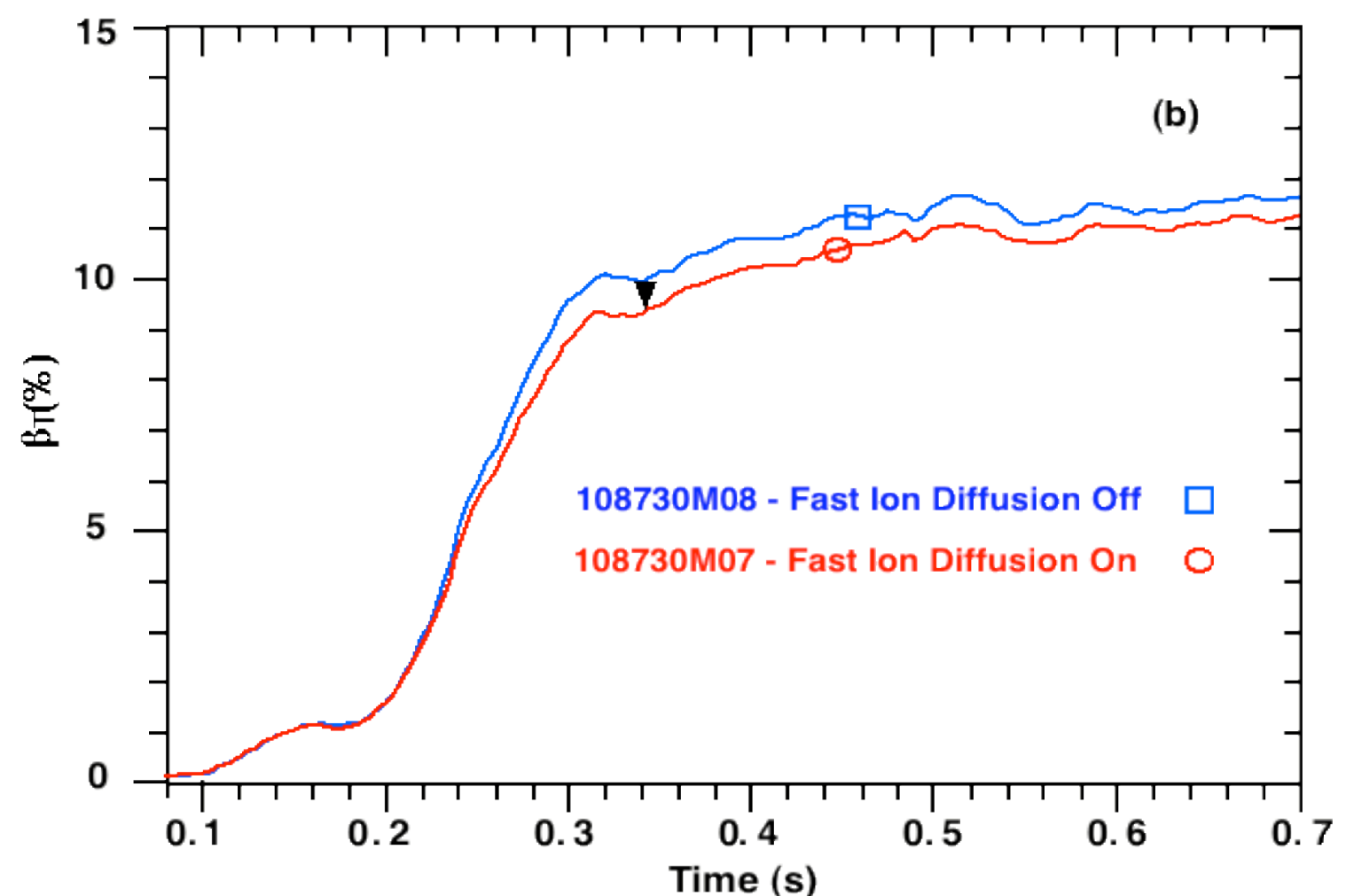

Figure 19. TRANSP calculation of thermal energy confinement time (a) and toroidal beta (b) at $t=400 \mathrm{~ms}$ with and without fast ion diffusion for SN108730 is shown. For this case, accounting for fast ion loss increased the thrmal energy confinement, $\left[\right.$, by $\sim 15 \%$ but reduced the toroidal beta, $\square_{T}$, by $\sim 7 \%$. 


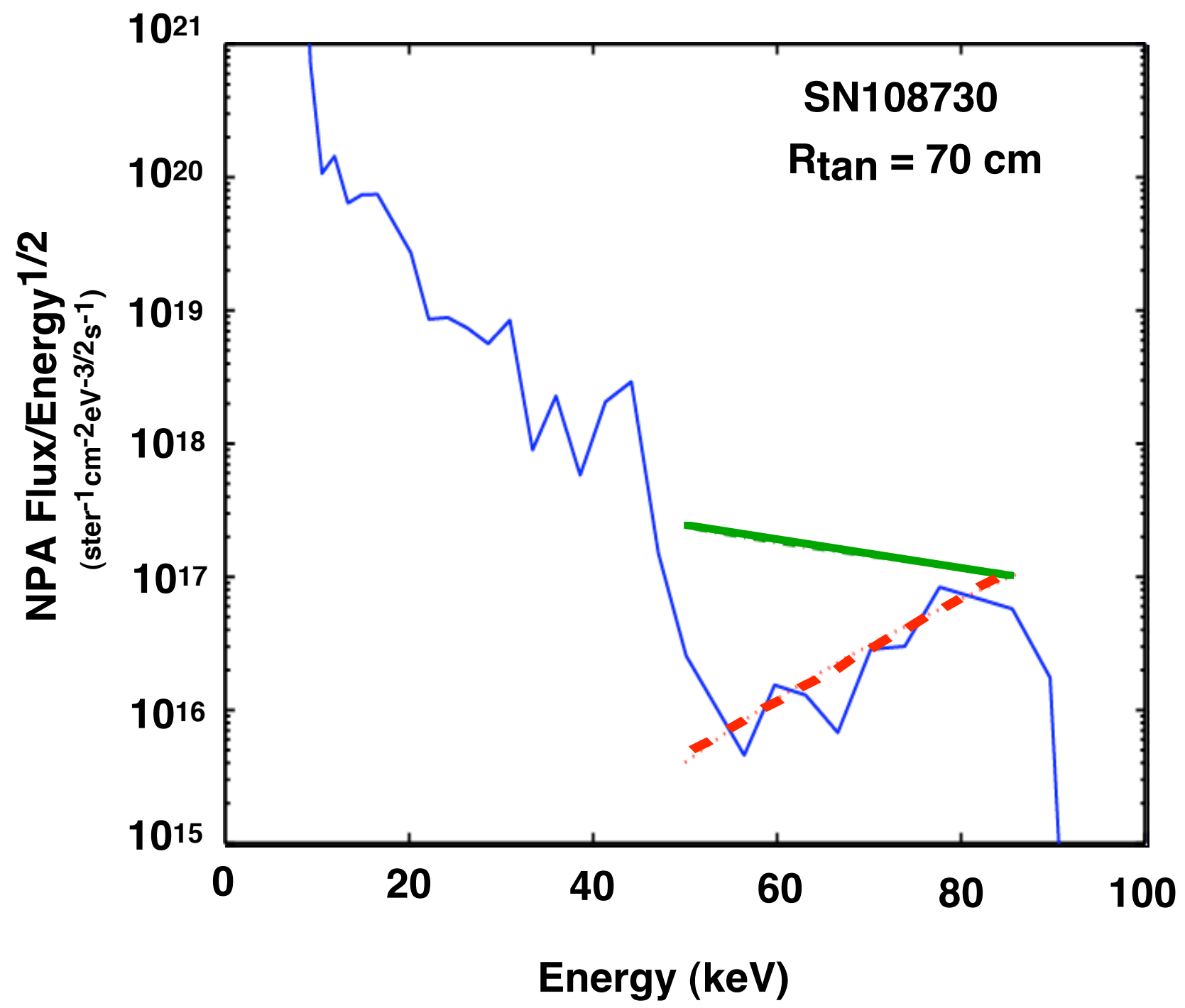

Figure 20. The NPA energetic ion spectrum and its approximation at high energy by the slowing down distribution without loss (solid line) and with finite loss time (dashed line) is shown. From analysis of the energy spectrum, the energetic ion loss time can be estimated to be on the order of $4 \mathrm{~ms}$. 


\section{External Distribution}

Plasma Research Laboratory, Australian National University, Australia

Professor I.R. Jones, Flinders University, Australia

Professor João Canalle, Instituto de Fisica DEQ/IF - UERJ, Brazil

Mr. Gerson O. Ludwig, Instituto Nacional de Pesquisas, Brazil

Dr. P.H. Sakanaka, Instituto Fisica, Brazil

The Librarian, Culham Laboratory, England

Mrs. S.A. Hutchinson, JET Library, England

Professor M.N. Bussac, Ecole Polytechnique, France

Librarian, Max-Planck-Institut für Plasmaphysik, Germany

Jolan Moldvai, Reports Library, Hungarian Academy of Sciences, Central Research Institute for Physics, Hungary

Dr. P. Kaw, Institute for Plasma Research, India

Ms. P.J. Pathak, Librarian, Institute for Plasma Research, India

Ms. Clelia De Palo, Associazione EURATOM-ENEA, Italy

Dr. G. Grosso, Instituto di Fisica del Plasma, Italy

Librarian, Naka Fusion Research Establishment, JAERI, Japan

Library, Laboratory for Complex Energy Processes, Institute for Advanced Study, Kyoto University, Japan

Research Information Center, National Institute for Fusion Science, Japan

Dr. O. Mitarai, Kyushu Tokai University, Japan

Dr. Jiangang Li, Institute of Plasma Physics, Chinese Academy of Sciences, People's Republic of China

Professor Yuping Huo, School of Physical Science and Technology, People's Republic of China

Library, Academia Sinica, Institute of Plasma Physics, People's Republic of China

Librarian, Institute of Physics, Chinese Academy of Sciences, People's Republic of China

Dr. S. Mirnov, TRINITI, Troitsk, Russian Federation, Russia

Dr. V.S. Strelkov, Kurchatov Institute, Russian Federation, Russia

Professor Peter Lukac, Katedra Fyziky Plazmy MFF UK, Mlynska dolina F-2, Komenskeho Univerzita, SK-842 15 Bratislava, Slovakia

Dr. G.S. Lee, Korea Basic Science Institute, South Korea

Institute for Plasma Research, University of Maryland, USA

Librarian, Fusion Energy Division, Oak Ridge National Laboratory, USA

Librarian, Institute of Fusion Studies, University of Texas, USA

Librarian, Magnetic Fusion Program, Lawrence Livermore National Laboratory, USA

Library, General Atomics, USA

Plasma Physics Group, Fusion Energy Research Program, University of California at San Diego, USA

Plasma Physics Library, Columbia University, USA

Alkesh Punjabi, Center for Fusion Research and Training, Hampton University, USA

Dr. W.M. Stacey, Fusion Research Center, Georgia Institute of Technology, USA

Dr. John Willis, U.S. Department of Energy, Office of Fusion Energy Sciences, USA

Mr. Paul H. Wright, Indianapolis, Indiana, USA 
The Princeton Plasma Physics Laboratory is operated by Princeton University under contract with the U.S. Department of Energy.

\author{
Information Services \\ Princeton Plasma Physics Laboratory \\ P.O. Box 451 \\ Princeton, NJ 08543
}

Phone: 609-243-2750

Fax: 609-243-2751

e-mail: pppl_info@pppl.gov

Internet Address: http://www.pppl.gov 